\title{
Wiener-Hopf Factorization of Transfer Functions of Extended Pritchard-Salamon Realizations
}

By M.A. KaAshoek of Amsterdam, C. V. M. VAN DER MeE of Cagliari, and A.C. M. RAN of Amsterdam

(Received February 26, 1998)

\begin{abstract}
In this paper factorization results for transfer functions of Pritchard-Salamon eystems are obtained. In particular, transfer functions sufficiently close to the identity operator are shown to have a canonical Wiener-Hopf factorization. Moreover, the Bounded Real Lemma is generalized to Pritchard-Salamon systems and applied to relate left and right canonical Wiener-Hopf factorizations of their transfer functions.
\end{abstract}

\section{Introduction}

In the theory of $H^{\infty}$-control and in particular in the description of all solutions of the Nehari problem one encounters the following problem (see [GGLD]). Given $W(\lambda)=G(-\bar{\lambda})^{*} J G(\lambda)$, where $G(\lambda)$ is a stable transfer function with a stable inverse, and $J=J^{*}=J^{-1}$, one seeks a factorization of the form $W(\lambda)=Z(-\bar{\lambda})^{*} J Z(\lambda)$, where $Z(\lambda)$ and its inverse are antistable. In other words, given a left canonical Wiener-Hopf factorization of $W(\lambda)$ one is looking for a right canonical Wiener-Hopf factorization of $W(\lambda)$.

In case the stable transfer function $G(\lambda)$ is rational, and thus the corresponding system is finite dimensional the unsymmetric version of this problem was solved in [BR], using methods from [BGK1]. A generalization to infinite dimensions in the context of realizations of the type considered in [BGK2] was given in [BC]. The symmetric version of the problem of left versus right factorization, with applications to the $\mathrm{Ne}$ hari problem, was studied in $[R]$ within a class of realizations with bounded input and output maps, a class smaller than the class studied in [BGK2]. (See also [CZ1] for this case.)

1991 Mathematics Subject Classification. Primary: 47 A68, Secondary: 93 A20, 93B28.

Keywords and phrases. Pritchard - Salamon systems, transfer functions, Wiener-Hopf factorization, Lyapunov equations, Riccati equations. 
This paper concerns the general problem of left versus right factorization for a class of infinite dimensional systems which has been introduced in [PS, S] and is known as the Pritchard-Salamon class (cf. [C, CLTZ, vK1, KMR]). Systems from this class have been succesfully used in the analysis of control and optimization problems involving partial differential equations and/or delay equations (see, e.g., [vK1], compare also [CZ1]). In [CR] the symmetric version of the problem was studied within the context of the class of realizations considered in the present paper, i. e., the Pritchard-Salamon class. In the latter paper, however, the input and output spaces were taken to be finite dimensional. In [CZ2] a factorization approach to the Nehari problem was given for the case where the input and output spaces are allowed to be infinite dimensional.

If $G(\lambda)$ is the transfer function of a stable Pritchard - Salamon system, then $W(\lambda)$ is not necessarily the transfer function of a stable Pritchard-Salamon system. This motivates the introduction of a generalization of the class of stable Pritchard-Salamon systems, as to encompass noncausal systems. The definition of these systems is given in terms of so - called extended Pritchard -Salamon realizations, where the noncausality forces one to employ bisemigroups (as introduced in [BGK2, BGK3]) instead of semigroups. This class of systems is introduced in Section 3, while some elementary properties of such systems are discussed in Section 4. We will draw heavily on [KMR] where basic results on the corresponding (causal) Pritchard-Salamon systems were derived and the class of functions that may occur as transfer functions of PritchardSalamon systems was described directly. Section 2 is of a preparatory nature and contains a review of the theory of bisemigroups.

The main result of this paper is a canonical Wiener-Hopf factorization theorem for transfer functions of extended Pritchard-Salamon (PS) realizations in terms of complementary pairs of invariant subspaces of the bigenerator of the bisemigroup governing the state space evolution of the system and that of the bisemigroup governing the state space evolution of the system obtained by reversing the roles of input and output. This result is presented in Section 5. Explicit formulas for the factors in a factorization that is canonical, apart from the fact that convergence at infinity is in the strong operator topology instead of the norm topology, are given in terms of the operators appearing in an extended PS - realization of the operator function under consideration. In particular, in case the transfer function of an extended PS-system is close to the identity operator it can be shown that these so-called quasi - canonical factorizations (both left and right) exist. For transfer functions of stable PS - systems the Bounded Real Lemma is derived. As in the finite dimensional case, it is shown that if $W(\lambda)$ is the transfer function of a stable PS-system and $\|W(\lambda)-I\|<1$ for real $\lambda$, then a certain algebraic operator Riccati equation has a solution, and conversely. All of these results exist for finite dimensional systems (see, e. g, [BGK1, GRa, GRu]); some of them exist for a class of infinite dimensional systems described by so-called BGK realizations [BGK2, BGK3] and for a class of infinite dimensional systems used to study abstract transport equations [GMP]. They are new for the class of extended Pritchard-Salamon realizations, with the exception of the Bounded Real Lemma for the class of stable PS-systems, which was derived earlier in [vK2], but for which we give a different proof.

In the final section we return to the problem outlined in the beginning of this introduction. Necessary and sufficient conditions are given for the existence of a right 
quasi - canonical factorization of the transfer funtion if this function is known to have a left quasi-canonical factorization.

We conclude this introduction with a few words about notation. We denote the open upper and lower half-planes by $\mathbb{C}^{+}$and $\mathbb{C}^{-}$, and their closures (including $\infty$ ) by $\overline{\mathbb{C}^{+}}$and $\overline{\mathbb{C}^{-}}$, respectively. If $X$ and $Y$ are complex Banach spaces, we denote by $\mathcal{L}(X, Y)$ the set of bounded linear operators from $X$ into $Y$; if $X=Y$ we write $\mathcal{L}(X)$ instead of $\mathcal{L}(X, X)$. The domain of an operator $T(X \rightarrow X)$ is denoted by $\mathcal{D}(T)$. Its kernel, range, resolvent set and spectrum are denoted by $\operatorname{Ker} T, \operatorname{Im} T, \rho(T)$ and $\sigma(T)$, respectively. For $1 \leq p \leq \infty,(E, \mu)$ a measure space and $Y$ a complex Banach space, $L_{p}(E ; Y)$ denotes the Banach space of all strongly measurable functions $f: E \rightarrow Y$ such that $\|f(\cdot)\|_{Y} \in L_{p}(E)$, endowed with the norm $\|f\|=\left[\int_{E}\|f(t)\|_{Y}^{p} d \mu(t)\right]^{p}$ if $1 \leq p<\infty$ and $\|f\|_{p}=\operatorname{ess~sup}_{t \in E}\|f(t)\|_{Y}$ if $p=\infty$ (cf. [DU]).

\section{Bisemigroups}

A strongly continuous semigroup $S(t)$ on a complex Banach space $X$ is called exponentially decaying if $\|S(t)\| \leq M e^{-\alpha t}$ for certain $M, \alpha>0$. If $A(X \rightarrow X)$ denotes its infinitesimal generator, i. e., $S(t)=e^{t A}$, then its spectrum $\sigma(A)$ is contained in the closed half-plane of all $\lambda$ with $\operatorname{Re} \lambda \leq-\alpha$.

Let $X$ be a complex Banach space and let $A(X \rightarrow X)$ be a linear operator defined on a linear subspace $\mathcal{D}(A)$ of $X$ with values in $X$. We say that $A$ is exponentially dichotomous if $A$ is densely defined (i.e., $\mathcal{D}(A)$ is dense in $X$ ) and $X$ admits a topological direct sum decomposition

$$
X=X_{-} \oplus X_{+}
$$

with the following properties: the decomposition reduces $A$, the restriction $-A_{-}$of $-A$ to $X_{-}$is the infinitesimal generator of an exponentially decaying semigroup, and the same is true for the restriction $A_{+}$of $A$ to $X_{+}$. The projection of $X$ onto $X_{-}$ along $X_{+}$is called a separating projection for $A$. This projection is uniquely determined by $A$. Now suppose $A(X \rightarrow X)$ is exponentially dichotomous, and let (2.1) be the decomposition having the properties described above. With respect to this decomposition, we write

$$
A=\left(\begin{array}{cc}
A_{-} & 0 \\
0 & A_{+}
\end{array}\right) \text {. }
$$

The bisemigroup $E(\cdot ; A)$ generated by $A$ is then defined as follows:

$$
E(t ; A) x= \begin{cases}-e^{t A}-P x, & t<0, \\ e^{t A}+(I-P) x, & t>0,\end{cases}
$$

where $P$ is the separating projection for $A$. The operator $A$ will sometimes be referred to as the bigenerator of $E(\cdot ; A)$. Note that the function $E(\cdot ; A)$ takes its values in $\mathcal{L}(X)$, the Banach space of all bounded linear operators on $X$. If $A$ is bounded, $A$ is exponentially dichotomous if and only if $\sigma(A)$ does not meet the imaginary axis, and then the separating projection is just the Riesz projection corresponding to the 
part of $\sigma(A)$ lying in the open right half-plane. A detailed theory of exponentially dichotomous operators may be found in [BGK2, BGK3].

Since $-A_{-}$and $A_{+}$generate exponentially decaying semigroups, there exists a constant $\omega>0$ such that

$$
\sup _{t \leq 0} e^{\omega t}\left\|e^{t A-}\right\|<\infty, \quad \sup _{t \geq 0} e^{-\omega t}\left\|e^{t A+}\right\|<\infty .
$$

If (2.3) is fulfilled, we say that $A$ (or the bisemigroup generated by $A$ ) is of exponential type $-\omega$. Note that (2.3) is equivalent to

$$
\sup _{t \neq 0} e^{\omega|t|}\|E(t ; A)\|<\infty .
$$

The infimum of all $-\omega$ such that $A$ is of exponential type $-\omega$ will be called the exponential growth bound of $A$ (or of the bisemigroup generated by $A$ ).

Recall (cf. [HP, P]) that the spectrum of the generator of an exponentially decaying semigroup is a closed subset of the set of all $\lambda$ with $\operatorname{Re} \lambda \leq-\omega$. As a result, if $A$ is exponentially dichotomous, then $\{\lambda \in \mathbb{C}:|\operatorname{Re} \lambda|<\omega\} \subset \rho(A)$ for some $\omega>0$.

For later use we mention a few simple facts about bisemigroups. Suppose $A(X \rightarrow X)$ is exponentially dichotomous, and let $E(\cdot ; A)$ be the corresponding bisemigroup given by (2.2). Take $x \in X$. The function $E(\cdot ; A) x$ is continuous on $\mathbb{R} \backslash\{0\}$ and Bochner integrable on $\mathbb{R}$. It also has a jump discontinuity at the origin and in fact

$$
E\left(0^{-} ; A\right) x:=\lim _{t \uparrow 0} E(t ; A) x=-P x, \quad E\left(0^{+} ; A\right) x:=\lim _{t \downarrow 0} E(t ; A) x=(I-P) x,
$$

where $P$ is the separating projection for $A$. If $x$ belongs to the domain $\mathcal{D}(A)$ of $A$, then $E(\cdot ; A) x$ is differentiable on $\mathbb{R} \backslash\{0\}$ and

$$
\frac{d}{d t} E(t ; A) x=E(t ; A) A x=A E(t ; A) x, \quad t \neq 0 .
$$

Obviously, for $x \in \mathcal{D}(A)$ the derivative of $E(\cdot ; A) x$ is continuous on $\mathbb{R} \backslash\{0\}$, Bochner integrable on $\mathbb{R}$ and has a jump discontinuity at the origin. From (2.2) it is clear that

$$
\begin{aligned}
E(t ; A) P & =P E(t ; A) & =E(t ; A), & t<0, \\
E(t ; A)(I-P) & =(I-P) E(t ; A) & =E(t ; A), & t>0 .
\end{aligned}
$$

Moreover, the following semigroup properties hold:

$$
E(t+s ; A)=\left\{\begin{aligned}
-E(t ; A) E(s ; A), & t, s<0, \\
E(t ; A) E(s ; A), & t, s>0 .
\end{aligned}\right.
$$

\section{Extended Pritchard - Salamon realizations: basic proper- ties}

Let $V$ and $W$ be complex Hilbert spaces (not necessarily separable), and let $\tau: W \rightarrow V$ be a fixed continuous and dense imbedding. For $A(V \rightarrow V)$ a possibly unbounded operator we define the part $A_{W}$ of $A$ in $W$ by

$$
\mathcal{D}\left(A_{W}\right)=\{x \in W: \tau x \in \mathcal{D}(A), A \tau x \in \tau[W]\}, \quad \tau\left(A_{W} x\right)=A \tau x, \quad x \in \mathcal{D}\left(A_{W}\right) .
$$


Then $A_{W}(W \rightarrow W)$ is a closed operator whenever $A(V \rightarrow V)$ is closed, but it may fail to be densely defined, even if $A(V \rightarrow V)$ is densely defined.

Let $Y$ be a complex Hilbert space. We call $\theta=(A, B, C ; V, W ; Y)$ an extended Pritchard-Salamon realization (or an extended PS-realization for short) if

1. $-i A(V \rightarrow V)$ is exponentially dichotomous;

2. The part $-i A_{W}$ of $-i A$ in $W$ is exponentially dichotomous and

$$
E(\cdot ;-i A) \tau=\tau E\left(\cdot ;-i A_{W}\right) ;
$$

3. $B \in \mathcal{L}(Y, V)$ and $C \in \mathcal{L}(W, Y)$;

4. There is a bounded linear operator $\Lambda_{\theta}: V \rightarrow L_{2}(\mathbb{R} ; Y)$ such that

$$
\Lambda_{\theta} \tau x=C E\left(\cdot ;-i A_{W}\right) x, \quad x \in W ;
$$

5. There is a bounded linear operator $\Gamma_{\theta}: L_{2}(\mathbb{R} ; Y) \rightarrow W$ such that

$$
\tau \Gamma_{\theta} \phi=\int_{-\infty}^{\infty} E(s ;-i A) B \phi(s) d s(\epsilon \tau[W]), \quad \phi \in L_{2}(\mathbb{R} ; Y) .
$$

Note that for every $t \in \mathbb{R}$ there is a bounded linear operator $\Gamma_{\theta, t}: L_{2}(\mathbb{R} ; Y) \rightarrow W$ such that

$$
\tau \Gamma_{\theta, t} \phi=\int_{-\infty}^{\infty} E(t-s ;-i A) B \phi(s) d s(\epsilon \tau[W]), \quad \phi \in L_{2}(\mathbb{R} ; Y),
$$

namely the operator satisfying

$$
\tau \Gamma_{\theta, t} \phi=\tau \Gamma_{\theta} \phi(t-\cdot), \quad \phi \in L_{2}(\mathbb{R} ; Y) .
$$

In [KMR] a definition of a Pritchard -Salamon realization $\theta$ appears that differs from the present definition of an extended Pritchard-Salamon realization in the following respects. In the first place $-i A(V \rightarrow V)$ and its part in $W$ are generators of strongly continuous semigroups instead of exponentially dichotomous operators. Moreover, these semigroups need not be exponentially decaying; in fact when they are, $\theta$ is called a stable Pritchard-Salamon realization. Finally, instead of $Y$ one has an input space $U$ and an output space $Y$ such that $B \in \mathcal{L}(U, V)$ and $C \in \mathcal{L}(W, Y)$, where $U$ and $Y$ may be different. Nevertheless, we have the following connection between extended PS - realizations and stable PS - realizations; its proof may be given by inspection.

Proposition 3.1. Let $\theta=(A, B, C ; V, W ; Y)$ be an extended $P S$-realization, and let $P$ and $P_{W}$ denote the separating projections of the bisemigroups $E(\cdot ;-i A)$ and $E\left(\cdot ;-i A_{W}\right)$, respectively. With respect to the decompositions of $V$ and $W$ as direct sums of the ranges $V_{-}$and $W_{-}$and the kernels $V_{+}$and $W_{+}$of $P$ and $P_{W}$, respectively, write

$$
A=\left(\begin{array}{cc}
A_{-} & 0 \\
0 & A_{+}
\end{array}\right), \quad B=\left(\begin{array}{l}
B_{-} \\
B_{+}
\end{array}\right), \quad C=\left(\begin{array}{cc}
C_{-} & C_{+}
\end{array}\right) .
$$

Then $\theta_{ \pm}=\left(\mp A_{ \pm}, B_{ \pm}, C_{ \pm} ; V_{ \pm}, W_{ \pm}, Y, Y\right)$ are stable $P S$-realizations. Conversely, let $\theta_{ \pm}=\left(\mp A_{ \pm}, B_{ \pm}, C_{ \pm} ; V_{ \pm}, W_{ \pm}, Y, Y\right)$ be stable $P S$-realizations, and define $V=V_{-} \oplus V_{+}$, 
$W=W_{-} \oplus W_{+}$, and $A, B$ and $C$ as in (3.2). Then $\theta=(A, B, C ; V, W ; Y)$ is an extended $P S$ - realization.

The definition of a PS - realization as given in [KMR] was inspired by the definition of a Pritchard-Salamon system as given in [PS, CLTZ]. In these works, as well as in the later [ $\mathrm{vK} 1$ ], the operator $\tau: W \rightarrow V$ is always the natural imbedding, which, as we have seen in [KMR], complicates the duality theory. Prior to [vK1], all references on the subject include the requirement $\mathcal{D}(A) \subset W$ in the definition of a PritchardSalamon system. Both in [vK1] and our previous paper [KMR] this class of systems was studied without this requirement.

The next lemma is a technical result immediate from Lemma 1.2 in [KMR] and Proposition 3.1.

Lemma 3.2. Let $\theta=(A, B, C ; V, W ; Y)$ be an extended $P S$ - realization, and let $-\omega_{\theta}$ be the maximum of the growth bounds of the bisemigroups $E(\cdot ;-i A)$ and $E\left(\cdot ;-i A_{W}\right)$. Then for every $\mu \in\left[0, \omega_{\theta}\right)$ there are constants $\gamma(\mu)$ and $\beta(\mu)$ such that

$$
\begin{array}{rlrl}
\left\|e^{\mu|\cdot|} \Lambda_{\theta} x\right\|_{L_{2}(\mathbb{R} ; Y)} & \leq \gamma(\mu)\|x\|_{V}, & & x \in V ; \\
\left\|\Gamma_{\theta}\left(e^{\mu|\cdot|} \phi\right)\right\|_{W} \leq \beta(\mu)\|\phi\|_{L_{2}(\mathbb{R} ; Y)}, & & \phi \in L_{2}(\mathbb{R} ; Y) .
\end{array}
$$

These statements are also valid if $L_{2}$ is replaced by $L_{1}$.

The number $-\omega_{\theta}$ defined in the lemma above plays an important role in the sequel, and will be used throughout without further explanation.

Making $\mathcal{D}(A)$ into a Hilbert space by endowing it with the graph norm $\|x\|_{\mathcal{D}(A)}=$ $\left[\|x\|_{V}^{2}+\|A x\|_{V}^{2}\right]^{1 / 2}$, we have (cf. Proposition 3.1, and [KMR], Proposition 1.4):

Proposition 3.3. Let $\theta=(A, B, C ; V, W ; Y)$ be an extended $P S$ - realization. Then there exists a unique $\bar{C}: \mathcal{D}(A) \rightarrow Y$ such that $C x=\bar{C} \tau x, x \in \mathcal{D}\left(A_{W}\right)$, and $\tilde{C}$ is $A$-bounded, i. e., $\tilde{C} \in \mathcal{L}(\mathcal{D}(A), Y)$ where $\mathcal{D}(A)$ is endowed with the graph norm $\|\cdot\|_{\mathcal{D}(A)}$.

Using $\tilde{C}: \mathcal{D}(A) \rightarrow Y$ as well as the bounded linear operators

$$
\hat{C}=\tilde{C} A^{-1}: V \rightarrow Y, \hat{B}=\tau^{-1} A^{-1} B: Y \rightarrow W,
$$

there are three equivalent ways of defining the transfer function of an extended PS realization $\theta=(A, B, C ; V, W ; Y)$ (cf. Proposition 3.1, and [KMR], Propositions 2.1 and 2.2), namely

$$
\begin{aligned}
W(\lambda) & =I+\tilde{C}(\lambda-A)^{-1} B \\
& =I+\hat{C} A(\lambda-A)^{-1} B \\
& =I+C A_{W}\left(\lambda-A_{W}\right)^{-1} \hat{B}
\end{aligned}
$$

where $|\operatorname{Im} \lambda|<\omega_{\theta}$. Note that, in contrast to [KMR], we have inserted the term $I$ in the definition of $W_{\theta}(\cdot)$. In other words, we always assume implicitly that the feedthrough 
coefficient of an extended PS-realization is equal to the identity operator $I$ on $Y$. The reason for this choice will become clear in Theorem 4.2.

From Proposition 3.3 and (3.5) we readily derive the following identities:

$$
\begin{aligned}
\left(\Lambda_{\theta} x\right)(t) & =\tilde{C} E(t ;-i A) x, & & x \in \mathcal{D}(A), \\
\left(\Lambda_{\theta} A^{-1} x\right)(t) & =\hat{C} E(t ;-i A) x, & & x \in V,
\end{aligned}
$$

$$
A_{W}^{-1} \Gamma_{\theta} \phi=\int_{-\infty}^{\infty} E\left(t ;-i A_{W}\right) \hat{B} \phi(t) d t, \quad \phi \in L_{2}(\mathbb{R} ; Y) .
$$

The weighting pattern of an extended PS-realization $\theta=(A, B, C ; V, W ; Y)$ is defined as (cf. [KMR])

$$
k_{\theta}: \mathbb{R} \longrightarrow \mathcal{L}(Y), \quad k_{\theta}(t) u=\left(\Lambda_{\theta} B u\right)(t) .
$$

From (3.3) and $B \in \mathcal{L}(Y, V)$ we immediately find for every $\mu \in\left[0, \omega_{\theta}\right)$ that

$$
\left\|e^{\mu|\cdot|} k_{\theta}(\cdot) u\right\|_{L_{2}(\mathbb{R} ; Y)} \leq \text { const. }\|u\|_{Y} .
$$

A similar estimate holds with $L_{2}$ replaced by $L_{1}$. In analogy with Eq. (1.5) of [KMR] using $I$ as the feedthrough coefficient, we now define the input-output operator by

$$
\left(T_{\theta} \phi\right)(t)=\phi(t)+(P) \int_{-\infty}^{\infty} k_{\theta}(t-s) \phi(s) d s, \quad t \in \mathbb{R} \quad \text { a.e. }
$$

where the integral is a Pettis integral [DU]. Observing that the Fourier transform

$$
\hat{\phi}(\lambda)=(\mathcal{F} \phi)(\lambda)=\int_{-\infty}^{\infty} e^{i \lambda t} \phi(t) d t
$$

is an invertible operator on $L_{2}(\mathbb{R} ; Y)$ such that $(2 \pi)^{-1 / 2} \mathcal{F}$ is unitary [DU], we have the following result (cf. Proposition 3.2, and [KMR], Corollary 2.3):

Proposition 3.4. Let $\theta=(A, B, C ; V, W ; Y)$ be an extended $P S$-realization. Then the input-output operator $T_{\theta}$ is a bounded linear operator on $L_{2}(\mathbb{R} ; Y)$ satisfying

$$
\left(\widehat{T_{\theta} \phi}\right)(\lambda)=W_{\theta}(\lambda) \hat{\phi}(\lambda), \quad \lambda \in \mathbb{R} .
$$

The following analogous result, however, requires a proof.

Proposition 3.5. Let $\theta=(A, B, C ; V, W ; Y)$ be an extended $P S$-realization. Then the operators $\mathcal{C}$ and $\mathcal{B}$ defined by

$$
\begin{aligned}
& \left(\mathcal{C} \phi_{l}\right)(t)=-i \int_{-\infty}^{\infty} \tilde{C} E(t-s ;-i A) \phi_{l}(s) d s \\
& \left(B \phi_{r}\right)(t)=-i \tau^{-1} \int_{-\infty}^{\infty} E(t-s ;-i A) B \phi_{r}(s) d s
\end{aligned}
$$


are bounded from $L_{2}(\mathbb{R} ; V)$ into $L_{2}(\mathbb{R} ; Y)$ and from $L_{2}(\mathbb{R} ; Y)$ into $L_{2}(\mathbb{R} ; W)$, respectively, and satisfy

$$
\left(\widehat{C \phi_{l}}\right)(\lambda)=\tilde{C}(\lambda-A)^{-1} \hat{\phi}_{l}(\lambda), \quad\left(\widehat{B}_{r}\right)(\lambda)=A_{W}\left(\lambda-A_{W}\right)^{-1} \hat{B} \hat{\phi}_{r}(\lambda)
$$

where $\hat{f}(\lambda)=\int_{-\infty}^{\infty} e^{i \lambda t} f(t) d t$.

Proof. Let us define the auxiliary bounded operators $\check{C}: L_{2}(\mathbb{R} ; W) \rightarrow L_{2}(\mathbb{R} ; Y)$ and $\check{\mathcal{B}}: L_{2}(\mathbb{R} ; Y) \rightarrow L_{2}(\mathbb{R} ; V)$ by the Bochner integrals

$$
\begin{aligned}
& \left(\check{\mathcal{C}} \psi_{l}\right)(t)=-i \int_{-\infty}^{\infty} C E\left(t-s ;-i A_{W}\right) \psi_{l}(s) d s, \\
& \left(\check{\mathcal{B}} \psi_{r}\right)(t)=-i \int_{-\infty}^{\infty} E(t-s ;-i A) B \psi_{r}(s) d s,
\end{aligned}
$$

where the boundedness is immediate from the exponential decay of the bisemigroups. By Fourier transformation we get

$$
\left(\widehat{\bar{C} \psi_{l}}\right)(\lambda)=C\left(\lambda-A_{W}\right)^{-1} \hat{\psi}_{l}(\lambda), \quad\left(\widehat{\mathcal{B} \psi_{r}}\right)(\lambda)=(\lambda-A)^{-1} B \hat{\psi}_{r}(\lambda)
$$

Now note that

$$
\begin{aligned}
\tilde{C}(\lambda-A)^{-1} \tau & =\hat{C} A(\lambda-A)^{-1} \tau=C\left(\lambda-A_{W}\right)^{-1}, \\
(\lambda-A)^{-1} B & =\tau A_{W}\left(\lambda-A_{W}\right)^{-1} \hat{B} .
\end{aligned}
$$

Further (cf. [GGK], p. 410), $A(\lambda-A)^{-1}$ and $A_{W}\left(\lambda-A_{W}\right)^{-1}$ vanish in the strong operator topology on $V$ and $W$ as $\lambda \rightarrow \pm \infty$ and hence are bounded in $\mathcal{L}(V)$ and $\mathcal{L}(W)$ for $\lambda \in \mathbb{R}$. Hence the premultiplication $M_{l}$ of a vector in $L_{2}(\mathbb{R} ; V)$ by $\bar{C}(\lambda-A)^{-1}$ is a bounded operator from $L_{2}(\mathbb{R} ; V)$ into $L_{2}(\mathbb{R} ; Y)$ and the premultiplication $M_{r}$ of a vector in $L_{2}(\mathbb{R} ; Y)$ by $A_{W}\left(\lambda-A_{W}\right)^{-1} \hat{B}$ is a bounded operator from $L_{2}(\mathbb{R} ; Y)$ into $L_{2}(\mathbb{R} ; W)$.

Now note that for every $t \in \mathbb{R}$ the right - hand sides of (3.11) and (3.12) are vectors in $Y$ and $W$ whose norms are bounded above by $\gamma(0)\left\|\phi_{l}\right\|_{L_{2}(\mathbb{R} ; V)}$ and $\beta(0)\left\|\phi_{r}\right\|_{L_{2}(\mathbb{R} ; Y)}$, respectively (cf. (3.3) - (3.4)). Further,

$$
\begin{aligned}
\left(\mathcal{F}^{-1} M_{l} \mathcal{F} \tau \psi_{l}\right)(t) & =\left(\mathcal{C} \tau \psi_{l}\right)(t)=\left(\check{\mathcal{C}} \psi_{l}\right)(t), & & \psi_{l} \in L_{2}(\mathbb{R} ; W) \\
\tau\left(\mathcal{F}^{-1} M_{r} \mathcal{F} \psi_{r}\right)(t) & =\tau\left(\mathcal{B} \psi_{r}\right)(t)=\left(\dot{\mathcal{B}} \psi_{r}\right)(t), & & \psi_{r} \in L_{2}(\mathbb{R} ; Y) .
\end{aligned}
$$

Consequently, $\mathcal{C}$ and $\mathcal{B}$ are bounded as claimed above.

Note that the operators $T_{\theta}, \mathcal{C}$ and $B$ satisfy the identity

$$
T_{\theta}=I+C B=I+C B,
$$

where $B$ is considered as a bounded linear operator $B: L_{2}(\mathbb{R} ; Y) \rightarrow L_{2}(\mathbb{R} ; V)$ and $C$ is considered as a bounded linear operator $C: L_{2}(\mathbb{R} ; W) \rightarrow L_{2}(\mathbb{R} ; Y)$. 


\section{Generating extended Pritchard-Salamon realizations}

In this section we construct, starting from two extended PS-realizations $\theta_{1}$ and $\theta_{2}$, an extended PS-realization $\theta$, called the product of $\theta_{1}$ and $\theta_{2}$, such that $W_{\theta}(\lambda)=$ $W_{\theta_{1}}(\lambda) W_{\theta_{2}}(\lambda)$ for $\lambda \in \mathbb{R}$. Next, given the extended PS-realization $\theta$ we define the extended PS-realizations $\theta^{\times}$and $\theta^{*}$, called the associate and the adjoint extended PS-realization, respectively, such that $W_{\theta \times}(\lambda)=W_{\theta}(\lambda)^{-1}$ and $W_{\theta \cdot}(\lambda)=W(\bar{\lambda})^{*}$ for $\lambda \in \mathbb{R}$.

We begin with the product of two extended PS-realizations.

Theorem 4.1. Let $\theta_{1}=\left(A_{1}, B_{1}, C_{1} ; V_{1}, W_{1} ; Y\right)$ and $\theta_{2}=\left(A_{2}, B_{2}, C_{2} ; V_{2}, W_{2} ; Y\right)$ be extended $P S$ - realizations. Put

$$
\begin{gathered}
\tau=\tau_{1} \oplus \tau_{2}, \quad V=V_{1} \oplus V_{2}, \quad W=W_{1} \oplus W_{2} ; \\
A=\left(\begin{array}{cc}
A_{1} & B_{1} \tilde{C}_{2} \\
0 & A_{2}
\end{array}\right), \quad B=\left(\begin{array}{c}
B_{1} \\
B_{2}
\end{array}\right), \quad C=\left(\begin{array}{ll}
C_{1} & C_{2}
\end{array}\right),
\end{gathered}
$$

where $\tilde{C}_{2}$ is defined as in Proposition 3.3. Then $\theta=(A, B, C ; V, W ; Y)$ is an extended $P S$-realization. Moreover,

$$
W_{\theta}(\lambda)=W_{\theta_{1}}(\lambda) W_{\theta_{2}}(\lambda), \quad|\operatorname{Im} \lambda|<\min \left(\omega_{\theta_{1}}, \omega_{\theta_{2}}\right) .
$$

The PS-realization $\theta$ as defined in the theorem is called the product of $\theta_{1}$ and $\theta_{2}$.

Proof. From Proposition 3.3 it is clear that $B_{1} \tilde{C}_{2}$ is an $A_{2}$-bounded operator into $V_{1}$, which makes $A$ with $\mathcal{D}(A)=\mathcal{D}\left(A_{1}\right) \oplus \mathcal{D}\left(A_{2}\right)$ well-defined.

Put .

$$
E(t)=\left(\begin{array}{cc}
E\left(t ;-i A_{1}\right) & F(t) \\
0 & E\left(t ;-i A_{2}\right)
\end{array}\right),
$$

where

$$
F(t) x=-i \int_{-\infty}^{\infty} E\left(t-s ;-i A_{1}\right) B_{1}\left(\Lambda_{\theta_{2}} x\right)(s) d s, \quad x \in V_{2},
$$

and

$$
E_{W}(t)=\left(\begin{array}{cc}
E\left(t ;-i A_{1, W}\right) & F_{W}(t) \\
0 & E\left(t ;-i A_{2, W}\right)
\end{array}\right)
$$

where

$$
F_{W}(t) x=-i \tau_{1}^{-1} \int_{-\infty}^{\infty} E\left(t-s ;-i A_{1}\right) B_{1} C_{2} E\left(s ;-i A_{2}, W\right) x d s, \quad x \in W_{2} .
$$

Then $\Lambda_{\theta_{2}} \in \mathcal{L}\left(V_{2}, L_{2}(\mathbb{R} ; Y)\right)$ implies that $F(t) \in \mathcal{L}\left(V_{2}, V_{1}\right), F_{W}(t) \in \mathcal{L}\left(W_{2}, W_{1}\right)$ and

$$
F(t) \tau_{2}=\tau_{1} F_{W}(t)
$$

and hence

$$
E(t) \tau=\tau E_{W}(t), \quad t \in \mathbb{R} \backslash\{0\}
$$


Next, for $\left(x_{1} x_{2}\right)^{T} \in V=V_{1} \oplus V_{2}$ we have

$$
\begin{aligned}
-i \int_{-\infty}^{\infty} e^{i \lambda t} E(t)\left(\begin{array}{l}
x_{1} \\
x_{2}
\end{array}\right) d t & =\left(\begin{array}{c}
\left(\lambda-A_{1}\right)^{-1} x_{1}+\left(\lambda-A_{1}\right)^{-1} B_{1} \tilde{C}_{2}\left(\lambda-A_{2}\right)^{-1} x_{2} \\
\left(\lambda-A_{2}\right)^{-1} x_{2}
\end{array}\right) \\
& =(\lambda-A)^{-1}\left(\begin{array}{c}
x_{1} \\
x_{2}
\end{array}\right) .
\end{aligned}
$$

Indeed, for $x_{2} \in \mathcal{D}\left(A_{2}\right)$ one can use (3.7) to derive this formula. As all three parts in the identity above are bounded in $V$, the identity holds for all $\left(\begin{array}{ll}x_{1} & x_{2}\end{array}\right)^{T} \in V$. Hence $E(\cdot)=E(\cdot ;-i A)$ is an exponentially decaying bisemigroup. Similarly, by applying (3.13) to $\theta_{1}$, we have for $\left(\begin{array}{ll}x_{1} & x_{2}\end{array}\right)^{T} \in W$

$$
\begin{aligned}
& -i \int_{-\infty}^{\infty} e^{i \lambda t} E_{W}(t)\left(\begin{array}{c}
x_{1} \\
x_{2}
\end{array}\right) d t \\
= & \left(\begin{array}{c}
\left(\lambda-A_{1, W}\right)^{-1} x_{1}+A_{1, W}\left(\lambda-A_{1, W}\right)^{-1} \hat{B}_{1} C_{2}\left(\lambda-A_{2, W}\right)^{-1} x_{2} \\
\left(\lambda-A_{2, W}\right)^{-1} x_{2}
\end{array}\right) \\
= & \left(\lambda-A_{W}\right)^{-1}\left(\begin{array}{c}
x_{1} \\
x_{2}
\end{array}\right),
\end{aligned}
$$

where the last equality follows with the help of (4.2). Indeed, denoting the identical left and middle members of the above equality by $G_{W}(\lambda)$, we see directly that $\tau\left[\operatorname{Im} G_{W}(\lambda)\right] \subset \mathcal{D}(A)$ and $(\lambda-A)^{-1} \tau=\tau G_{W}(\lambda)$, so that $G_{W}(\lambda)$ is the resolvent of the part $A_{W}$ of $A$ in $W$. Hence $E_{W}(\cdot)=E\left(\cdot ;-i A_{W}\right)$ is an exponentially decaying bisemigroup.

Next, we compute $\left(\Lambda_{\theta}\left(\begin{array}{l}x_{1} \\ x_{2}\end{array}\right)\right)(t)$ for $\left(\begin{array}{ll}x_{1} & x_{2}\end{array}\right)^{T} \in V$. Taking Fourier transforms, using (3.10) for $\theta_{1}$, and (3.13) for $\theta_{1}$ and $\theta_{2}$, and then taking inverse Fourier transforms we compute that

$$
\left(\Lambda_{\theta}\left(\begin{array}{l}
x_{1} \\
x_{2}
\end{array}\right)\right)(t)=\left(\Lambda_{\theta_{1}} x_{1}\right)(t)+\left(\Lambda_{\theta_{2}} x_{2}\right)(t)+(P) \int_{-\infty}^{\infty} k_{\theta_{1}}(t-s)\left(\Lambda_{\theta_{2}} x_{2}\right)(s) d s,
$$

where the integral is to be understood as a Pettis integral. Hence by Proposition 3.4

$$
\left\|\Lambda_{\theta}\left(\begin{array}{c}
x_{1} \\
x_{2}
\end{array}\right)\right\|_{L_{2}(\mathbb{R} ; Y)} \leq \text { const. }\left(\left\|x_{1}\right\|_{V_{1}}^{2}+\left\|x_{2}\right\|_{V_{2}}^{2}\right)^{1 / 2}=\text { const. }\left\|\left(\begin{array}{c}
x_{1} \\
x_{2}
\end{array}\right)\right\|_{V} .
$$

By computing $\tau \Gamma_{\theta} \phi$ we also find

$$
\Gamma_{\theta} \phi=\left(\begin{array}{c}
\Gamma_{\theta_{1}} \phi+\Gamma_{\theta_{1}} \psi \\
\Gamma_{\theta_{2}} \phi
\end{array}\right)
$$

where in terms of a Pettis integral

$$
\psi(t)=\left(T_{\theta_{2}} \phi\right)(t)=(P) \int_{-\infty}^{\infty} k_{\theta_{2}}(t-s) \phi(s) d s, \quad t \in \mathbb{R} .
$$

Hence, for all $\phi \in L_{2}(\mathbb{R} ; Y)$

$$
\left\|\Gamma_{\theta} \phi\right\|_{W} \leq \text { const. }\left(\|\phi\|_{L_{2}\left(\mathbb{R}_{;} Y\right)}+\|\psi\|_{L_{2}(\mathbb{R} ; Y)}\right) \leq \text { const. }\|\phi\|_{L_{2}(\mathbb{R} ; Y)} .
$$


Finally, (4.1) follows from the first line of (3.6).

Let $\theta=(A, B, C ; V, W ; Y)$ be an extended $\mathrm{PS}$ - realization. Put $A^{\times}=A-B \tilde{C}$ viewed as an operator from $\mathcal{D}(A)$ to $V$. Then for $\lambda \notin \sigma\left(A^{\times}\right) \cup \sigma(A)$ the operator $W_{\theta}(\lambda)$ is invertible and we have

$$
W_{\theta}(\lambda)^{-1}=I-\tilde{C}\left(\lambda-A^{\times}\right)^{-1} B
$$

Indeed, this is an easy consequence of the fact that $B \tilde{C}$ may be written as $\left(\lambda-A^{\times}\right)-$ $(\lambda-A)$. Put $\theta^{\times}=\left(A^{\times}, B,-C ; V, W ; Y\right)$. The next theorem shows that under certain additional conditions $\theta^{\times}$is an extended PS - realization and that its transfer function is $W_{\theta}(\lambda)^{-1}$ for $\lambda \in \mathbb{R}$.

Theorem 4.2. Let $\theta=(A, B, C ; V, W ; Y)$ be an extended $P S$-realization, and put $A^{\times}=A-B \tilde{C}$. Suppose there exist $\nu \in\left(0, \omega_{\theta}\right)$ and $M>0$ such that $W_{\theta}(\lambda)$ is invertible on $Y$ with $\left\|W_{\theta}(\lambda)^{-1}\right\| \leq M$ if $|\operatorname{Im} \lambda| \leq \nu$. Then $\theta^{\times}=\left(A^{\times}, B,-C ; V, W ; Y\right)$ is an extended $P S$ - realization, and

$$
W_{\theta \times}(\lambda)=W_{\theta}(\lambda)^{-1}, \quad \lambda \in \mathbb{R} .
$$

Proof. Note that $\mathcal{D}\left(A^{\times}\right)=\mathcal{D}(A)$. Let $A_{W}^{\times}$be the part of $A^{\times}$in $W$. Consider the full line convolution equation

$$
H(t) x+(P) \int_{-\infty}^{\infty} k_{\theta}(t-s) H(s) x d s=-i\left(\Lambda_{\theta} x\right)(t), \quad t \in \mathbb{R} \backslash\{0\},
$$

where $x \in V$ and the integral is to be understood as a Pettis integral. Then for every $x \in V$

$$
H(t) x=\frac{1}{2 \pi} \int_{-\infty}^{\infty} e^{-i \lambda t} W_{\theta}(\lambda)^{-1} \tilde{C}(\lambda-A)^{-1} x d \lambda, \quad t \in \mathbb{R},
$$

defines the unique solution of (4.4) in $L_{2}(\mathbb{R} ; Y)$. Because $e^{\mu|\cdot|} \Lambda_{\theta} x \in L_{2}(\mathbb{R} ; Y)$ for every $x \in V$ [cf. (3.3)] and $\left\|W_{\theta}(\lambda)^{-1}\right\| \leq M$ if $|\operatorname{Im} \lambda| \leq \nu\left(<\omega_{\theta}\right)$, we have $e^{\nu|\cdot|} H(\cdot) x \in L_{2}(\mathbb{R} ; Y)$ and

$$
\left\|e^{\nu|\cdot|} H(\cdot) x\right\|_{L_{2}(\mathbb{R} ; Y)} \leq \text { const. }\left\|e^{\nu|\cdot|} \Lambda_{\theta} x\right\|_{L_{2}(\mathbb{R} ; Y)} \leq \text { const. }\|x\|_{V}, \quad x \in V .
$$

Next, put

$$
S(t) x=E(t ;-i A) x-\int_{-\infty}^{\infty} E(t-s ;-i A) B H(s) x d s, \quad t \in \mathbb{R} \backslash\{0\},
$$

for $x \in V$, and

$$
S_{W}(t) x=E\left(t ;-i A_{W}\right) x-\tau^{-1} \int_{-\infty}^{\infty} E(t-s ;-i A) B H(s) \tau x d s, \quad t \in \mathbb{R} \backslash\{0\},
$$

for $x \in W$. Then $S(\cdot) x \in L_{2}(\mathbb{R} ; V)$ for $x \in V, S_{W}(\cdot) x \in L_{2}(\mathbb{R} ; W)$ for $x \in W$ [cf. Proposition 3.5], and

$$
S(t) \tau=\tau S_{W}(t), \quad t \in \mathbb{R} \backslash\{0\} .
$$


From (3.4) and (4.5) we get

$$
\left\|S_{W}(t) x\right\|_{W} \leq \text { const. } e^{-\nu|t|}\|x\|_{W} .
$$

By Fourier transformation, for $x \in V$

$$
\begin{aligned}
-i \int_{-\infty}^{\infty} e^{i \lambda t} S(t) x d t & =(\lambda-A)^{-1} x-(\lambda-A)^{-1} B \hat{H}(\lambda) x \\
& =(\lambda-A)^{-1} x-(\lambda-A)^{-1} B W_{\theta}(\lambda)^{-1} \tilde{C}(\lambda-A)^{-1} x \\
& =\left(\lambda-A^{\times}\right)^{-1} x,
\end{aligned}
$$

whereas for $x \in W$

$$
\begin{aligned}
& -i \int_{-\infty}^{\infty} e^{i \lambda t} S_{W}(t) x d t \\
= & \left(\lambda-A_{W}\right)^{-1} x-A_{W}\left(\lambda-A_{W}\right)^{-1} \hat{B} W_{\theta}(\lambda)^{-1} C\left(\lambda-A_{W}\right)^{-1} x \\
= & \left(\lambda-A_{W}^{\times}\right)^{-1} x .
\end{aligned}
$$

To justify the last equality, we denote the equivalent left and middle members of (4.7) by $K_{W}(\lambda)$ and find $\tau K_{W}(\lambda)=\left(\lambda-A^{\times}\right)^{-1} \tau$, so that $K_{W}(\lambda)$ is the resolvent of the part $A_{W}^{\times}$of $A^{\times}$in $W$. Hence, $-i A^{\times}$and $-i A_{W}^{\times}$are exponentially dichotomous, $S(t)=E\left(t ;-i A^{\times}\right)$, and $S_{W}(t)=E\left(t ;-i A_{W}^{x}\right)$.

Now observe that for every $x \in W$

$$
\Lambda_{\theta \times} \tau x+\Lambda_{\theta} x=i C B H(\cdot) \tau x
$$

where $C$ is to be read as an operator from $L_{2}(\mathbb{R} ; W)$ into $L_{2}(\mathbb{R} ; Y)$. Then the boundedness of $C$ and $\Lambda_{\theta},(4.5)$, and Proposition 3.5 imply

$$
\left\|\Lambda_{\theta \times x}\right\|_{L_{2}\left(\mathbb{R}_{i} Y\right)} \leq \text { const. }\|x\|_{V}, \quad x \in V .
$$

Next, observe [cf. (4.4) and (3.12)]

$$
E\left(t ;-i A^{\times}\right) x-E(t ;-i A) x=-i \tau(\mathcal{B} H(\cdot) x)(t), \quad x \in V .
$$

Then, by Proposition 3.5 and (4.5),

$$
e^{\nu|\cdot|}\|B H(\cdot) B u\|_{L_{2}(\mathbb{R} ; W)} \leq \text { const. }\|u\|_{\gamma},
$$

so that

$$
\|\mathcal{B} H(\cdot) B u\|_{L_{1}(\mathbb{R} ; W)} \leq \text { const. }\|u\|_{Y} .
$$

This implies, by (3.13),

$$
C_{H}:=\sup _{\lambda \in \mathbb{R}}\left\|A_{W}\left(\lambda-A_{W}\right)^{-1} \hat{B} \hat{H}(\lambda) B\right\|_{\mathcal{L}(Y, W)}<+\infty .
$$

Now, for $\phi \in L_{2}(\mathbb{R} ; Y)$ we obtain from (4.6)

$$
\Gamma_{\theta \times \phi}-\Gamma_{\theta} \phi=-\tau^{-1} \int_{-\infty}^{\infty} \tau^{-1} \int_{-\infty}^{\infty} E(t-s ;-i A) B H(s) B \phi(t) d s d t .
$$


With the help of (4.8) we then get

$$
\left\|\Gamma_{\theta \times} \phi-\Gamma_{\theta} \phi\right\| W \leq C_{H}\|\phi\|_{L_{2}(\mathbb{R} ; Y)} .
$$

As a result, $\theta^{\times}=\left(A^{\times}, B,-C ; V, W ; Y\right)$ is an extended PS-realization.

Corollary 4.3. Let $\theta=(A, B, C ; V, W ; Y)$ be an extended $P S$-realization, and put

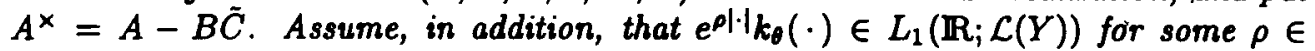
$\left(0, \omega_{\theta}\right)$. Then $\left(A^{\times}, B,-C ; V, W ; Y\right)$ is an extended $P S$-realization if $\sigma\left(A^{\times}\right) \cap \mathbb{R}=\emptyset$.

Proof. Under the additional hypothesis on $k_{\theta}$, we have

$$
\lim _{\lambda \rightarrow \infty,|\operatorname{Im} \lambda| \leq \rho}\left\|W_{\theta}(\lambda)-I\right\|=0 .
$$

From $\sigma\left(A^{\times}\right) \cap \mathbb{R}=\emptyset$ it follows, taking into account the observation made before Theorem 4.2, that $W_{\theta}(\lambda)$ is invertible for real $\lambda$. Combining these facts one sees that there exist $\nu \in(0, \rho] \subset\left(0, \omega_{\theta}\right)$ and $M>0$ such that $W_{\theta}(\lambda)$ is invertible and $\left\|W_{\theta}(\lambda)^{-1}\right\| \leq M$ if $|\operatorname{Im} \lambda| \leq \nu$.

The extra assumption on $k_{\theta}$ made in Corollary 4.3 is always satisfied if $Y$ is a finite-dimensional space (see [BGK2]).

Finally, we define the adjoint $\theta^{*}$ of an extended PS-realization $\theta$. The adjoint of a Pritchard-Salamon system was defined before in [vK1]. However, the use of the natural imbedding of $W$ into $V$ in this adjoint realization coupled to the use of the same Hilbert state spaces $V$ and $W$ for the adjoint system led to a rather complicated definition. A more natural approach was the way in which the adjoint $\theta^{*}$ of a PSrealization $\theta$ was defined in [KMR]. The realization studied in [KMR] has the symmetry properties

$$
k_{\theta} \cdot(t)=k_{\theta}(t)^{*}, \quad W_{\theta} \cdot(\lambda)=W_{\theta}(-\bar{\lambda}),
$$

reflecting the emphasis put on the weighting pattern. Below we will modify the definition of the adjoint PS - realization given in [KMR] to account for bisemigroups and for the more convenient symmetry relations

$$
k_{\theta *}(t)=k_{\theta}(-t)^{*}, \quad W_{\theta} \cdot(\lambda)=W_{\theta}(\bar{\lambda}),
$$

because in the present paper the transfer function is emphasized instead of the weighting pattern. The crucial idea underlying the definitions of $\theta^{*}$ in [KMR] and in the present paper is the reversal of the roles of the state spaces $V$ and $W$ and the use of the adjoint operator $\tau^{*}: V \rightarrow W$ as the imbedding of $V$ into $W$.

Theorem 4.4. Let $\theta=(A, B, C ; V, W ; Y)$ be an extended $P S$-realization. Then the six-tuple $\theta^{*}=\left(A_{W}^{*}, C^{*}, B^{*} ; W, V ; Y\right)$ is also an extended $P S$-realization and

$$
k_{\theta \cdot}(t)=k_{\theta}(-t)^{*}, \quad W_{\theta \cdot}(\lambda)=W_{\theta}(\bar{\lambda}) \text {. }
$$

The proof of Theorem 4.4 is very similar to the proof of Proposition 3.1 of [KMR]. The major changes are the use of bisemigroups instead of semigroups and some sign 
differences due to the different symmetry relations in the weighting pattern and the transfer function.

We will list some of the properties of $\theta^{*}$ below. For their proofs we refer to [KMR] where some differences appear due to the different definitions of the adjoint.

(a) $\left(\tau^{*}\right)^{*}=\tau$.

(b) The part of $A_{W}^{*}(W \rightarrow W)$ in $V$ is $A^{*}(V \rightarrow V)$.

(c) $E\left(t ;-i A_{W}^{*}\right)=E\left(t ;-i A_{W}\right)^{*}$ and $E\left(t ;-i A^{*}\right)=E(t ;-i A)^{*}$.

(d) $\Lambda_{\theta^{*}}=\left(\Gamma_{\theta}\right)^{*}$ and $\Gamma_{\theta^{*}}=\left(\Lambda_{\theta}\right)^{*}$.

(e) Defining $\widehat{B^{*}}$ and $\widehat{C^{*}}$ in terms of $\theta^{*}$ as $\hat{C}$ and $\hat{B}$ have been specified in terms of $\theta$ in (3.5), we have

$$
\widehat{B^{*}}=(\hat{B})^{*}, \quad \widehat{C^{*}}=(\hat{C})^{*}
$$

\section{Factorization theorems}

In this section we study factorizations of transfer functions of extended PS - realizations where the factors and their inverses are analytic in the upper and lower halfplane and have strong limits at infinity. These factorizations will be called left and right quasi - canonical. We also generalize the Bounded Real Lemma on the existence of solutions of certain Riccati equations to extended Pritchard-Salamon realizations (see also [vK2]).

Let $Y$ be a complex Hilbert space. Suppose $W$ is an operator function defined on the extended real line with values in $\mathcal{L}(Y)$, which is continuous in the norm on $\mathbb{R}$ and strongly continuous at infinity. Then

$$
W(\lambda)=W_{+}(\lambda) W_{-}(\lambda), \quad \lambda \in \mathbb{R} \cup\{\infty\},
$$

is called a left quasi-canonical factorization of $W$ if

1. $W_{ \pm}$extends to an operator function that is continuous in the norm on $\mathbb{C}^{ \pm} \cup \mathbb{R}$, analytic on $\mathbb{C}^{ \pm}$, and strongly continuous on $\overline{\mathbb{C}^{ \pm}}$;

2. $W_{ \pm}(\lambda)$ is boundedly invertible for all $\lambda \in \overline{\mathbb{C}^{ \pm}}$;

3. $W_{ \pm}(\cdot)^{-1}$ is strongly continuous on $\overline{\mathbb{C}^{ \pm}}$.

The factorization is called a canonical factorization (instead of a quasi-canonical factorization) if the continuity in conditions 1 and 3 is with respect to the norm topology instead of the strong topology. In particular, in case $\operatorname{dim} Y<\infty$ the two notions coincide.

A factorization of $W$ of the form

$$
W(\lambda)=W_{-}(\lambda) W_{+}(\lambda), \quad \lambda \in \mathbb{R} \cup\{\infty\},
$$

where the factors $W_{ \pm}$have the properties $1-3$ stated above, is called a right quasicanonical factorization of $W$.

We begin with a general factorization result for transfer functions.

Theorem 5.1. Let $\theta=(A, B, C ; V, W ; Y)$ be an extended $P S$-realization such that

$$
W_{\theta}(\lambda)=I+\bar{C}(\lambda-A)^{-1} B=I+C A_{W}\left(\lambda-A_{W}\right)^{-1} \hat{B}
$$


is boundedly invertible and $\left\|W_{\theta}(\lambda)^{-1}\right\| \leq M$ if $|\operatorname{Im} \lambda| \leq \nu$. Let $A^{\times}=A-B \tilde{C}$ be defined on $\mathcal{D}(A)$. Suppose

$$
V_{-} \oplus V_{+}^{\times}=V, \quad W_{-} \oplus W_{+}^{\times}=W,
$$

where $V_{ \pm}=\operatorname{Im} E\left(0^{ \pm} ;-i A\right), W_{ \pm}=\operatorname{Im} E\left(0^{ \pm} ;-i A_{W}\right), V_{ \pm}^{\times}=\operatorname{Im} E\left(0^{ \pm} ;-i A^{\times}\right)$and $W_{ \pm}^{\times}=\operatorname{Im} E\left(0^{ \pm} ;-i A_{W}^{\times}\right)$. Let $A_{1}, A_{2}, A_{1}^{\times}$and $A_{2}^{\times}$be the parts of $A, A, A^{\times}$and $A^{\times}$in $V_{-}, V_{+}, V_{-}^{\times}$and $V_{+}^{\times}$, respectively, and let $A_{1, W}, A_{2, W}, A_{1, W}^{\times}$and $A_{2, W}^{\times}$be the parts of $A_{W}, A_{W}, A_{W}^{\times}$and $A_{W}^{\times}$in $W_{-}, W_{+}, W_{-}^{\times}$and $W_{+}^{\times}$, respectively. If $\Pi$ and $\Pi_{W}$ denote the projections of $V$ onto $V_{+}^{\times}$along $V_{-}$, respectively, the projection of $W$ onto $W_{+}^{\times}$along $W_{-}$, put $B_{1}=(I-\Pi) B: Y \rightarrow V_{-}, B_{2}=\Pi B: Y \rightarrow V_{+}^{\times}$, $C_{1}=C\left(I-\Pi_{W}\right): W_{-} \rightarrow Y$ and $C_{2}=C \Pi_{W}: W_{+}^{\times} \rightarrow Y$. Then

$$
\begin{aligned}
& \theta_{1}=\left(A_{1}, B_{1}, C_{1} ; V_{-}, W_{-} ; Y\right), \theta_{2}=\left(A_{2}, B_{2}, C_{2} ; V_{+}, W_{+} ; Y\right), \\
& \theta_{1}^{\times}=\left(A_{1}^{\times}, B_{1},-C_{1} ; V_{-}, W_{-} ; Y\right), \quad \theta_{2}^{\times}=\left(A_{2}^{\times}, B_{2},-C_{2} ; V_{+}, W_{+} ; Y\right)
\end{aligned}
$$

are extended $P S$ - realizations satisfying

$$
W_{\theta}(\lambda)=W_{\theta_{1}}(\lambda) W_{\theta_{2}}(\lambda), \quad|\operatorname{Im} \lambda| \leq \nu
$$

and

$$
W_{\theta}(\lambda)^{-1}=W_{\theta_{2}^{x}}(\lambda) W_{\theta_{1}^{x}}(\lambda), \quad|\operatorname{Im} \lambda| \leq \nu .
$$

Proof. First note that all of the parts of operators mentioned in the statement of Theorem 5.1 are densely defined. Observe also that $V_{-}$is $A$-invariant, $W_{-}$is $A_{W}$-invariant, $V_{+}^{\times}$is $A^{\times}$-invariant, and $W_{+}^{\times}$is $A_{W}^{\times}$-invariant. Remark also that if $\hat{\tau}_{1}$ and $\hat{\tau}_{2}$ are the natural imbeddings of $W_{1}$ into $W$ and of $W_{2}^{x}$ into $W$, then $\tau_{1}=\tau \hat{\tau}_{1}: W_{1} \rightarrow V_{1}$ and $\tau_{2}=\tau \hat{\tau}_{2}: W_{2}^{\times} \rightarrow V_{2}^{\times}$are continuous and dense imbeddings.

Define

$$
\begin{aligned}
E_{1}(t) & =E(t ;-i A)(I-\Pi), & E_{1}^{\times}(t) & =(I-\Pi) E\left(t ;-i A^{\times}\right)(I-\Pi), \\
E_{2}(t) & =\Pi E(t ;-i A) \Pi, & E_{2}^{\times}(t) & =E\left(t ;-i A^{\times}\right) \Pi, \\
E_{1, W}(t) & =E\left(t ;-i A_{W}\right)\left(I-\Pi_{W}\right), & E_{1, W}^{\times}(t) & =\left(I-\Pi_{W}\right) E\left(t ;-i A_{W}^{\times}\right)\left(I-\Pi_{W}\right), \\
E_{2, W}(t) & =\Pi_{W} E(t ;-i A) \Pi_{W}, & E_{2, W}^{\times}(t) & =E\left(t ;-i A_{W}^{\times}\right) \Pi_{W},
\end{aligned}
$$

where the operators $E_{1}(t)$ and $E_{1}^{\times}(t)$ act on $V_{1}$, the operators $E_{2}(t)$ and $E_{2}^{\times}(t)$ act on $V_{2}^{\times}$, the operators $E_{1, W}(t)$ and $E_{1, W}^{\times}(t)$ act on $W_{1}$, and finally, the operators $E_{2, W}(t)$ and $E_{2, W}^{\times}(t)$ act on $W_{2}^{\times}$. Then all eight operators $\mathcal{E}(t)$ given by (5.3) satisfy

$$
\|\mathcal{E}(t)\| \leq \text { const. } e^{-\nu|t|}, \quad t \in \mathbb{R} \backslash\{0\}
$$

and are strongly continuous in $t$ with a jump discontinuity at $t=0$. Moreover, for $r=1,2$

$$
\begin{aligned}
-i \int_{-\infty}^{\infty} e^{i \lambda t} E_{r}(t) d t & =\left(\lambda-A_{r}\right)^{-1}, \quad-i \int_{-\infty}^{\infty} e^{i \lambda t} E_{r}^{\times}(t) d t=\left(\lambda-A_{r}^{\times}\right)^{-1} \\
-i \int_{-\infty}^{\infty} e^{i \lambda t} E_{r, W}(t) d t & =\left(\lambda-A_{r, W}\right)^{-1}, \quad-i \int_{-\infty}^{\infty} e^{i \lambda t} E_{r, W}^{\times}(t) d t=\left(\lambda-A_{r, W}^{\times}\right)^{-1} .
\end{aligned}
$$


Thus all eight expressions in (5.3) define exponentially decaying bisemigroups. We will henceforth write them in terms of their bigenerators.

Next, we prove the identity

$$
\Pi \tau=\tau \Pi_{\boldsymbol{W}} .
$$

Indeed, taking $x \in W$ and writing $\Pi_{W} x=E\left(0^{+} ;-i A_{W}^{x}\right) w_{1}$ for some $w_{1} \in W$ and $\left(I-\Pi_{W}\right) x=E\left(0^{-} ;-i A_{W}\right) w_{2}$ for some $w_{2} \in W$, we find $\tau \Pi_{W} x=E\left(0^{+} ;-i A^{\times}\right) \tau w_{1} \in$ $\operatorname{Im} \Pi \tau$ and $\tau\left(I-\Pi_{W}\right) x=E\left(0^{-} ;-i A\right) \tau w_{2} \in \operatorname{Im}(I-\Pi) \tau$. Thus $\tau \Pi_{W} \tau^{-1}$ is a projection defined on $\tau[W]$ whose range and kernel are contained in the range and kernel of $\Pi$, respectively, and which is a restriction of $\Pi$. We then readily find (5.4).

Using (5.4) we have for $x \in W_{1}$

$$
\begin{aligned}
& \Lambda_{\theta_{1}} \tau_{1} x=C_{1} E\left(\cdot ;-i A_{1, W}\right) x=C\left(I-\Pi_{W}\right) E\left(\cdot ;-i A_{W}\right)\left(I-\Pi_{W}\right) x \\
& =C E\left(\cdot ;-i A_{W}\right)\left(I-\Pi_{W}\right) x=\Lambda_{\theta} \tau\left(I-\Pi_{W}\right) x \\
& =\Lambda_{\theta}(I-\Pi) \tau x=\Lambda_{\theta} x \text {, }
\end{aligned}
$$

so that $\Lambda_{\theta_{1}} \in \mathcal{L}\left(V_{1}, L_{2}(\mathbb{R} ; Y)\right)$. Similarly, for $x \in W_{2}$ we have

$$
\begin{aligned}
& \Lambda_{\theta_{2}^{\times} \tau_{2} x}=-C_{2} E\left(\cdot ;-i A_{2, W}^{\times}\right) x=-C \Pi_{W} E\left(\cdot ;-i A_{2, W}^{\times}\right) \Pi_{W} x \\
& =-C E\left(\cdot ;-i A_{W}^{x}\right) \Pi_{W} x=\Lambda_{\theta \times} \tau \Pi_{W} x \\
& =\Lambda_{\theta \times} \Pi \tau x=\Lambda_{\theta \times} \tau x \text {, }
\end{aligned}
$$

so that $\Lambda_{\theta_{2}^{\times}} \in \mathcal{L}\left(V_{2}, L_{2}(\mathbb{R} ; Y)\right)$. Using (5.4) we have for $\phi \in L_{2}(\mathbb{R} ; Y)$

$$
\begin{aligned}
\tau_{2} \Gamma_{\theta_{2}} \phi & =\int_{-\infty}^{\infty} E\left(s ;-i A_{2}\right) B_{2} \phi(s) d s=\int_{-\infty}^{\infty} \Pi E(s ;-i A) \Pi B \phi(s) d s \\
& =\Pi \int_{-\infty}^{\infty} E(s ;-i A) B \phi(s) d s=\Pi \tau \Gamma_{\theta} \phi \\
& =\tau \Pi_{W} \Gamma_{\theta} \phi
\end{aligned}
$$

so that $\Gamma_{\theta_{2}} \in \mathcal{L}\left(L_{2}(\mathbb{R} ; Y), W_{2}\right)$. Similarly, for $\phi \in L_{2}(\mathbb{R} ; Y)$ we have

$$
\begin{aligned}
\tau_{1} \Gamma_{\theta_{1}^{\times}} \phi & =\int_{-\infty}^{\infty} E\left(s ;-i A_{1}^{\times}\right) B_{1} \phi(s) d s \\
& =\int_{-\infty}^{\infty}(I-\Pi) E\left(s ;-i A^{\times}\right)(I-\Pi) B \phi(s) d s \\
& =(I-\Pi) \int_{-\infty}^{\infty} E\left(s ;-i A^{\times}\right) B \phi(s) d s \\
& =(I-\Pi) \tau \Gamma_{\theta \times} \phi \\
& =\tau\left(I-\Pi \Pi_{W}\right) \Gamma_{\theta \times} \phi,
\end{aligned}
$$

so that $\Gamma_{\theta_{1}^{\times}} \in \mathcal{L}\left(L_{2}(\mathbb{R} ; Y), W_{1}\right)$ 
The other four boundedness proofs for $\Lambda$ and $\Gamma$ operators are more involved. First, we find from (3.15) and (4.3)

$$
\begin{aligned}
\left(\lambda-A_{W}^{\times}\right)^{-1}-\left(\lambda-A_{W}\right)^{-1} & =-A_{W}\left(\lambda-A_{W}\right)^{-1} \hat{B} W_{\theta}(\lambda)^{-1} C\left(\lambda-A_{W}\right)^{-1} \\
& =-A_{W}\left(\lambda-A_{W}\right)^{-1} \hat{B} W_{\theta}(\lambda)^{-1} \tilde{C}(\lambda-A)^{-1} \tau \\
& =-A_{W}\left(\lambda-A_{W}\right)^{-1} \hat{B} \tilde{C}\left(\lambda-A^{\times}\right)^{-1}
\end{aligned}
$$

Similarly, we find using (3.15) and (4.3)

$$
\begin{aligned}
\tau\left[\left(\lambda-A_{W}^{\times}\right)^{-1}-\left(\lambda-A_{W}\right)^{-1}\right] & =-\tau A_{W}\left(\lambda-A_{W}\right)^{-1} \hat{B} W_{\theta}(\lambda)^{-1} C\left(\lambda-A_{W}\right)^{-1} \\
& =-(\lambda-A)^{-1} B W_{\theta}(\lambda)^{-1} C\left(\lambda-A_{W}\right)^{-1} \\
& =-\left(\lambda-A^{\times}\right)^{-1} B C\left(\lambda-A_{W}\right)^{-1} \\
& =-\tau A_{W}^{\times}\left(\lambda-A_{W}^{\times}\right)^{-1} \hat{B}^{\times} C\left(\lambda-A_{W}\right)^{-1}
\end{aligned}
$$

where $\hat{B}^{\times}=\tau^{-1}\left(A_{W}^{\times}\right)^{-1} B \in \mathcal{L}(Y, W)$. Hence

$$
\left(\lambda-A_{W}^{\times}\right)^{-1}-\left(\lambda-A_{W}\right)^{-1}=-A_{W}^{\times}\left(\lambda-A_{W}^{\times}\right)^{-1} \hat{B}^{\times} C\left(\lambda-A_{W}\right)^{-1} .
$$

Thus (5.5), (3.7) and (3.13) imply

$$
\left[E\left(t ;-i A_{W}^{\times}\right)-E\left(t ;-i A_{W}\right)\right] x=\left(B \Lambda_{\theta \times} \tau x\right)(t), \quad x \in W,
$$

where $\mathcal{B}$ is as in Proposition 3.5. Analogously, (5.6), (3.7) and (3.13) imply

$$
\left[E\left(t ;-i A_{W}^{\times}\right)-E\left(t ;-i A_{W}\right)\right] x=-\left(\mathcal{B}^{\times} \Lambda_{\theta} \tau x\right)(t), \quad x \in W,
$$

where $\left(\mathcal{B}^{\times} \phi\right)(t)=-i \tau^{-1} \int_{-\infty}^{\infty} E\left(t-s ;-i A^{\times}\right) B \phi(s) d s$ defines a bounded operator from $L_{2}(\mathbb{R} ; Y)$ into $W$. Hence for $x \in W_{1}$ we have

$$
\begin{aligned}
\Lambda_{\theta_{1}^{\times}} \tau_{1} x & =-C_{1} E\left(\cdot ;-i A_{1, W}^{\times}\right) x \\
& =-C\left(I-\Pi_{W}\right) E\left(\cdot ;-i A_{W}^{\times}\right)\left(I-\Pi_{W}\right) x \\
& =-C E\left(\cdot ;-i A_{W}\right)\left(I-\Pi_{W}\right) x-C\left(I-\Pi_{W}\right) \mathcal{B} \Lambda_{\theta \times} \tau\left(I-\Pi_{W}\right) x \\
& =-\Lambda_{\theta}(I-\Pi) \tau x-C\left(I-\Pi_{W}\right) \mathcal{B} \Lambda_{\theta \times}(I-\Pi) \tau x,
\end{aligned}
$$

so that $\Lambda_{\theta_{1}^{\times}} \in \mathcal{L}\left(V_{1} ; L_{2}(\mathbb{R} ; Y)\right)$. By the same token, for $x \in W_{2}$ we have

$$
\begin{aligned}
\Lambda_{\theta_{2}} \tau_{2} x & =C_{2} E\left(\cdot ;-i A_{2, W}\right) x \\
& =C \Pi_{W} E\left(\cdot ;-i A_{W}\right) \Pi_{W} x \\
& =C E\left(\cdot ;-i A_{W}^{\times}\right) \Pi_{W} x+C \Pi_{W} \mathcal{B}^{\times} \Lambda_{\theta \times} \Pi_{\tau x} \\
& =-\Lambda_{\theta}(I-\Pi) \tau x+C \Pi_{W} \mathcal{B}^{\times} \Lambda_{\theta \times} \Pi_{\tau x},
\end{aligned}
$$

so that $\Lambda_{\theta_{2}} \in \mathcal{L}\left(V_{2} ; L_{2}(\mathbb{R} ; Y)\right)$. 
The last two boundedness proofs for $\Gamma$ operators require the use of $\left(J_{Y} \phi\right)(t)=\phi(-t)$ on $L_{2}(\mathbb{R} ; Y)$ and $\left(J_{V} \psi\right)(t)=\psi(-t)$ on $L_{2}(\mathbb{R} ; V)$, as well as repeated use of (3.7) (3.9). Furthermore, we need the following two analogues of (5.5) and (5.6):

$$
\begin{aligned}
\left(\lambda-A^{\times}\right)^{-1}-(\lambda-A)^{-1} & =-(\lambda-A)^{-1} B \bar{C}\left(\lambda-A^{\times}\right)^{-1} \\
& =-\left(\lambda-A^{\times}\right)^{-1} B \bar{C}(\lambda-A)^{-1} .
\end{aligned}
$$

Using (5.7), (3.7) applied to $\theta^{\times}$, and (3.14) we then get

$$
\left[E\left(t ;-i A^{\times}\right)-E(t ;-i A)\right] x=\left(\hat{\mathcal{B}} \Lambda_{\theta \times} x\right)(t), \quad x \in V,
$$

while (5.7), (3.7), and the boundedness of the linear operator $\hat{\mathcal{B}}^{\times}$defined by $\left(\hat{\mathcal{B}}^{\times} \phi\right)(t)=$ $-i \int_{-\infty}^{\infty} E\left(t-s ;-i A^{\times}\right) B \phi(s) d s$ from $L_{2}(\mathbb{R} ; Y)$ into $L_{2}(\mathbb{R} ; V)$ (cf. (3.14) applied to $\left.\theta^{\times}\right)$imply

$$
\left[E\left(t ;-i A^{\times}\right)-E(t ;-i A)\right] x=-\left(\hat{\mathcal{B}}^{\times} \Lambda_{\theta} x\right)(t), \quad x \in V .
$$

For $\phi \in L_{2}(\mathbb{R} ; Y)$ we have

$$
\begin{aligned}
& \int_{-\infty}^{\tau_{1} \Gamma_{\theta_{1}} \phi}(I-\Pi)\left[E\left(s ;-i A^{\times}\right)-i \int_{-\infty}^{\infty} E\left(s-t ;-i A^{\times}\right) B \tilde{C} E(t ;-i A) d t\right](I-\Pi) B \phi(s) d s \\
= & (I-\Pi)\left\{\tau \Gamma_{\theta \times} \phi-i \int_{-\infty}^{\infty} \int_{-\infty}^{\infty} E\left(\sigma ;-i A^{\times}\right) B \tilde{C} E(s-\sigma ;-i A)(I-\Pi) B \phi(s) d s d \sigma\right\} \\
= & \tau\left(I-\Pi_{W}\right) \Gamma_{\theta \times} \phi+\tau\left(I-\Pi_{W}\right) \Gamma_{\theta \times} J_{Y} C J_{V}(I-\Pi) B \phi,
\end{aligned}
$$

so that $\Gamma_{\theta_{1}} \in \mathcal{L}\left(L_{2}(\mathbb{R} ; Y), W_{1}\right)$. For $\phi \in L_{2}(\mathbb{R} ; Y)$ we have

$$
\begin{aligned}
\tau_{2} \Gamma_{\theta_{2}^{\times}} \phi & =\int_{-\infty}^{\infty} \Pi\left[E(s ;-i A)+i \int_{-\infty}^{\infty} E(s-t ;-i A) B \tilde{C} E\left(t ;-i A^{\times}\right) d t\right] \Pi B \phi(s) d s \\
& =\Pi \tau \Gamma_{\theta} \phi+i \Pi \int_{-\infty}^{\infty} \int_{-\infty}^{\infty} E(\sigma ;-i A) B \tilde{C} E\left(s-\sigma ;-i A^{\times}\right) \Pi B \phi(s) d s d \sigma \\
& =\tau \Pi_{W} \Gamma_{\theta} \phi+\tau \Pi_{W} \Gamma_{\theta} J_{Y} \mathcal{C}^{\times} J_{V} \Pi B \phi,
\end{aligned}
$$

where $\left(\mathcal{C}^{\times} \phi\right)(t)=i \int_{-\infty}^{\infty} \tilde{C} E\left(t-s ;-i A^{\times}\right) \phi(s) d s$ defines a bounded operator from $L_{2}(\mathbb{R} ; V)$ into $L_{2}(\mathbb{R} ; Y)$ (cf. (3.12) applied to $\left.\theta^{\times}\right)$, so that $\Gamma_{\theta_{2}^{\times}} \in \mathcal{L}\left(L_{2}(\mathbb{R} ; Y), W_{2}\right)$. This completes the proof, as (5.1) and (5.2) follow from Theorem 4.1.

Theorem 5.2. Let $\theta=(A, B, C ; V, W ; Y)$ be an extended $P S$ - realization, and let $W_{\theta}(\cdot)^{-1}$ be uniformly bounded on the strip $|\operatorname{Im} \lambda| \leq \nu$ for some $\nu \in\left(0, \omega_{\theta}\right)$. Then the following statements are equivalent:

1. $W_{\theta}(\cdot)$ has a left quasi-canonical factorization; 
2. $V=V_{-} \oplus V_{+}^{\times}$and $W=W_{-} \oplus W_{+}^{\times}$, where $V_{-}, V_{+}^{\times}, W_{-}$and $W_{+}^{\times}$are defined in Theorem 5.1;

3. For every $g \in L_{2}\left(\mathbb{R}^{+} ; Y\right)$, the following equation is uniquely solvable in $L_{2}\left(\mathbb{R}^{+} ; Y\right)$ :

$$
\phi(t)+(P) \int_{0}^{\infty} k_{\theta}(t-s) \phi(s) d s=g(t), \quad t>0 ;
$$

4. Put $k_{l}(\cdot) x=-i B\left(\Lambda_{\theta} x\right)(\cdot)$ for $x \in V$. Then for every $g_{l} \in L_{2}\left(\mathbb{R}^{+} ; V\right)$, the following equation is uniquely solvable in $L_{2}\left(\mathbb{R}^{+} ; V\right)$ :

$$
\phi_{l}(t)+(P) \int_{0}^{\infty} k_{l}(t-s) \phi_{l}(s) d s=g_{l}(t), \quad t>0
$$

5. For $\psi \in L_{2}\left(\mathbb{R}^{+} ; W\right)$, put $(P) \int_{0}^{\infty} k_{r}(t) \psi(t) d t=-i \Gamma_{\theta} C \psi$. Then for every $g_{r} \in L_{2}\left(\mathbb{R}^{+} ; W\right)$, the following equation is uniquely solvable in the Hilbert space $L_{2}\left(\mathbb{R}^{+} ; W\right)$ :

$$
\phi_{r}(t)+(P) \int_{0}^{\infty} k_{r}(t-s) \phi_{r}(s) d s=g_{r}(t), \quad t>0 .
$$

In (5.8) - (5.10) the integrals are to be understood as Pettis integrals.

Proof. (2) $\Longrightarrow$ (1). This is the content of Theorem 5.1 .

$(3) \Longleftrightarrow(4) \Longleftrightarrow(5)$. Using Proposition 3.3 we write $(5.8)-(5.10)$ in the form

$$
\begin{array}{rlrl}
\phi+C B^{+} \phi & =\phi+C^{+} B \phi=g & \left(\in L_{2}\left(\mathbb{R}^{+} ; Y\right)\right), \\
\phi_{l}+B C^{+} \phi_{l} & =g_{l} & & \left(\in L_{2}\left(\mathbb{R}^{+} ; V\right)\right), \\
\phi_{r}+\mathcal{B}^{+} C \phi_{r} & =g_{r} & & \left(\in L_{2}\left(\mathbb{R}^{+} ; W\right)\right),
\end{array}
$$

where $\mathcal{C}^{+}$and $\mathcal{B}^{+}$are defined as $\mathcal{C}$ and $B$ but with the integration over $\mathbb{R}^{+}$. Since $\mathcal{C}^{+}: L_{2}\left(\mathbb{R}^{+} ; V\right) \rightarrow L_{2}\left(\mathbb{R}^{+} ; Y\right), \mathcal{B}^{+}: L_{2}\left(\mathbb{R}^{+} ; Y\right) \rightarrow L_{2}\left(\mathbb{R}^{+} ; W\right), B: L_{2}\left(\mathbb{R}^{+} ; Y\right) \rightarrow$ $L_{2}\left(\mathbb{R}^{+} ; V\right)$ and $C: L_{2}\left(\mathbb{R}^{+} ; W\right) \rightarrow L_{2}\left(\mathbb{R}^{+} ; Y\right)$ are bounded, these three equations are uniquely solvable if one of them is uniquely solvable.

$(1) \Longrightarrow(3)$. If $W$ has the left quasi-canonical factorization

$$
W_{\theta}(\lambda)=W_{-}(\lambda) W_{+}(\lambda), \quad|\operatorname{Im} \lambda| \leq \nu,
$$

then (5.8) reduces to the Riemann - Hilbert problem

$$
W_{+}(\lambda) \hat{\phi}_{+}(\lambda)+W_{-}(\lambda)^{-1} \hat{\phi}_{-}(\lambda)=W_{-}(\lambda)^{-1} \hat{g}(\lambda), \quad \lambda \in \mathbb{R},
$$

where

$$
\hat{\phi}_{ \pm}(\lambda)= \pm \int_{0}^{ \pm \infty} e^{i \lambda t} \phi(t) d t, \quad \hat{g}(\lambda)=\int_{0}^{\infty} e^{i \lambda t} g(t) d t,
$$

and $\phi(t)=-(P) \int_{0}^{\infty} k_{\theta}(t-s) \phi(s) d s$ for $t<0$, the integral being understood as a Pettis integral. From the unique additive decomposition

$$
W_{-}(\lambda)^{-1} \hat{g}(\lambda)=\hat{h}_{+}(\lambda)+\hat{h}_{-}(\lambda)
$$


with $\hat{h}_{ \pm}(\lambda)= \pm \int_{0}^{ \pm \infty} e^{i \lambda t} h(t) d t$ for some $h \in L_{2}(\mathbb{R} ; Y)$, we find the unique solution

$$
\phi(t)=\frac{1}{2 \pi} \int_{-\infty}^{\infty} e^{-i \lambda t} W_{+}(\lambda)^{-1} \hat{h}_{+}(\lambda) d \lambda, \quad t>0,
$$

in $L_{2}\left(\mathbb{R}^{+} ; Y\right)$.

$(5) \Longrightarrow(2)$. Consider the vector - valued integral equation

$$
G(t) x-i \tau^{-1} \int_{0}^{\infty} E(t-s ;-i A) B C G(s) x d s=E\left(t ;-i A_{W}\right) x, \quad t>0,
$$

where $x \in W$. By our hypothesis its unique solution $G(\cdot) x$ belongs to $L_{2}\left(\mathbb{R}^{+} ; W\right)$. For all $u \geq 0$ and $t>0$ we have

$$
\begin{aligned}
& \tau G(t+u) x-i \int_{0}^{\infty} E(t-s ;-i A) B C G(s+u) x d s \\
= & \tau G(t+u) x-i \int_{u}^{\infty} E(t+u-s ;-i A) B C G(s) x d s \\
= & \tau E\left(t+u ;-i A_{W}\right) x+i \int_{0}^{u} E(t+u-s ;-i A) B C G(s) x d s \\
= & \tau E\left(t ;-i A_{W}\right)\left[E\left(u ;-i A_{W}\right) x+i \tau^{-1} \int_{0}^{u} E(u-s ;-i A) B C G(s) x d s\right] \\
= & \tau E\left(t ;-i A_{W}\right)\left[E\left(u ;-i A_{W}\right) x+i \tau^{-1} \int_{0}^{\infty} E(u-s ;-i A) B C G(s) x d s\right] \\
= & \tau E\left(t ;-i A_{W}\right) G(u) x,
\end{aligned}
$$

where we used in the one but last equality the fact that $E(t ;-i A) E(s ;-i A)=0$ for $t>0$ and $s<0$. Thus

$$
G(t+u) x=G(t) G(u) x, \quad x \in W .
$$

As $G(\cdot)$ is strongly continuous from the right at zero, we see that $G(0)$ is a bounded projection on $W$. Further, if $x \in W$, we have $G(0) x=0$ iff $G(t) x \equiv 0$ iff $E\left(t ;-i A_{W}\right) x \equiv$ 0 iff $x \in W_{-}$, so that $\operatorname{Ker} G(0)=W_{-}$.

Let us substitute $E\left(\cdot ;-i A_{W}^{\times}\right) G(0) x$ with $x \in W$ in the left-hand side of (5.12). Taking Fourier transforms we find for the left - hand side of (5.12) the vector function $\hat{e}_{+}(\cdot)$, where $\hat{e}_{ \pm}(\lambda)= \pm \int_{0}^{ \pm \infty} e^{i \lambda t} e(t) d t$ with $e \in L_{2}\left(\mathbb{R}^{+} ; W\right)$ and

$$
\hat{e}_{+}(\lambda)+\hat{e}_{-}(\lambda)=\int_{-\infty}^{\infty} e^{i \lambda t} e(t) d t=\left(\lambda-A_{W}\right)^{-1}\left(\lambda-A_{W}^{\times}\right) \cdot\left(\lambda-A_{W}^{\times}\right)^{-1} G(0) x .
$$

Hence,

$$
\hat{e}_{+}(\lambda)=\left(\lambda-A_{W}\right)^{-1}\left(I-P_{W}\right) G(0) x,
$$

which equals $\left(\lambda-A_{W}\right)^{-1}\left(I-P_{W}\right) x$ because of $\operatorname{Ker} G(0)=W_{-}$. Thus $W_{-} \oplus \operatorname{Im} G(0)=$ $W$ and $\operatorname{Im} G(0) \subset W_{+}^{\times}$. Similarly, substituting $E\left(\cdot ;-i A_{W}^{\times}\right) x$ with $x \in W_{+}$in the left hand side of (5.12), we find $\left(I-P_{W}\right) E\left(\cdot ;-i A_{W}\right) x$ for the right-hand side. Thus $W_{-}+W_{+}^{\times}=W$. Finally, because of the unique solvability of (5.12) we obtain

$$
W_{-} \oplus W_{+}^{x}=W \text {, }
$$


so that $G(0)$ is the projection of $W$ onto $W_{+}^{\times}$along $W_{-}$.

Taking $t \downarrow 0$ in (5.12) we get for every $x \in W$

$$
\begin{aligned}
G(0) x & =\left(I-P_{W}\right) x+i \tau^{-1} \int_{0}^{\infty} E(-s ;-i A) B C E\left(s ;-i A_{W}^{x}\right) G(0) x d s \\
& =\left(I-P_{W}\right) x+J_{Y} \Gamma_{\theta} \Lambda_{\theta} \tau G(0) x,
\end{aligned}
$$

where $\left(J_{Y} \phi\right)(t)=\phi(-t)$, and therefore

$$
\left\|G(0) x-\left(I-P_{W}\right) x\right\|_{W} \leq \text { const. }\left\|\Lambda_{\theta} \tau G(0) x\right\|_{L_{2}\left(\mathbb{R}^{+} ; Y\right)} \leq \text { const. }\|\tau x\|_{V} .
$$

Thus there exists a bounded operator $Q$ on $V$ such that

$$
(Q-(I-P)) \tau x=\tau\left(G(0)-\left(I-P_{W}\right)\right) x, \quad x \in W
$$

Hence $V=V_{-} \oplus V_{+}^{\times}$and $Q$ is the projection of $V$ onto $V_{+}^{\times}$along $V_{-}$.

Analogues of the following corollary have been proved for norm continuous operator functions belonging to certain operator algebras [GL] and for the transfer function of the linear system naturally occurring in abstract transport theory [GMP].

Corollary 5.3. Let $\theta=(A, B, C ; V, W ; Y)$ be an extended $P S$ - realization, and let

$$
\sup _{|\operatorname{Im} \lambda| \leq \nu}\left\|W_{\theta}(\lambda)-I\right\|_{\mathcal{L}(Y)}<1
$$

Then $W_{\theta}(\cdot)$ has a left and a right quasi-canonical factorization.

Proof. Let $H_{ \pm}$be the closed subspaces of $L_{2}(\mathbb{R} ; Y)$ consisting of those $\hat{h}(\cdot)$ which can be written as $\hat{h}(\lambda)= \pm \int_{0}^{ \pm \infty} e^{i \lambda t} h(t) d t$ for some $h \in L_{2}\left(\mathbb{R}^{ \pm} ; Y\right)$, and let $\pi_{0}: L_{2}(\mathbb{R} ; Y) \rightarrow H_{+}$and $\tau_{0}: H_{+} \rightarrow L_{2}(\mathbb{R} ; Y)$ be defined in such a way that $\pi_{0} \tau_{0}$ is the identity operator on $H_{+}$and $\tau_{0} \pi_{0}$ is the orthogonal projection of $L_{2}(\mathbb{R} ; Y)$ onto $H_{+}$. Then (5.8) can be written in the equivalent form

$$
\hat{\phi}_{+}+\pi_{0}\left[W_{\theta}(\cdot)-I\right] \tau_{0} \hat{\phi}_{+}=\hat{g}
$$

where $\hat{\phi}_{+}$and $\hat{g}$ are given by (5.11) and belong to $H_{+}$. Since $\pi_{0}$ and $\tau_{0}$ have unit norm,

$$
\left\|\pi_{0}\left[W_{\theta}(\cdot)-I\right] \tau_{0}\right\|_{H_{+}} \leq \sup _{\lambda \in \mathbb{R}}\left\|W_{\theta}(\lambda)-I\right\|_{\mathcal{L}(Y)}<1
$$

and hence $(5.8)$ is uniquely solvable in $L_{2}\left(\mathbb{R}^{+} ; Y\right)$. As a result, $W_{\theta}$ has a left quasicanonical factorization.

The existence of a right quasi-canonical factorization follows by applying the same argument to $\xi=(-A, B,-C ; V, W ; Y)$, knowing that $W_{\xi}(\lambda)=W_{\theta}(-\lambda)$.

We note that Theorems 5.1 and 5.2 have counterparts involving right quasi - canonical factorization. This is also the case for Theorem 5.4 below, which generalizes the Bounded Real Lemma on the existence of solutions of Riccati equations. In [vK2] this 
result was derived as a consequence of a result on general LQ-optimal control problems for the class of PS - systems. The proof given below is based on our factorization theorems above.

Theorem 5.4. Let $\theta=(A, B, C ; V, W ; Y)$ be an extended $P S$-realization, and let $W_{\theta}(\cdot)^{-1}$ be uniformly bounded on the strip $|\operatorname{Im} \lambda| \leq \nu$. Suppose $\sigma(A) \cup \sigma\left(A_{W}\right) \subset \mathbb{C}_{-}$. Then

$$
\sup _{|\operatorname{Im} \lambda| \leq \nu_{0}}\left\|W_{\theta}(\lambda)-I\right\|<1
$$

for some $\nu_{0}>0$ if and only if the algebraic Riccati equation

$$
-i X A x+i A_{W}^{*} X x+X B \widetilde{B^{*}} X x+C^{*} \tilde{C} x=0, \quad x \in \mathcal{D}(A),
$$

has a solution $X \in \mathcal{L}(V, W)$ where $X[\mathcal{D}(A)] \subset \mathcal{D}\left(A_{W}^{*}\right)$,

$$
\sigma\left(A+i B \widetilde{B^{*}} X\right) \subset \mathbb{C}_{-}, \quad \sigma\left(A_{W}^{*}-i X B \widetilde{B^{*}}\right) \subset \mathbb{C}_{+},
$$

and $X \tau=\tau^{*} X_{V W}$ for some $X_{V W} \in \mathcal{L}(W, V)$. Here $\widetilde{B}^{*}$ is given in terms of the extended PS-realization $\theta^{*}$ by Proposition 3.3. Moreover, $X \tau \in \mathcal{L}(W)$ is selfadjoint.

Proof. We split the proof into four parts.

Part (a). Introduce $\psi=(\breve{A}, \breve{B}, \breve{C} ; \breve{V}, \breve{W} ; Y)$ where $\breve{V}=V \oplus W, \breve{W}=W \oplus V$, $\check{\tau}=\tau \oplus \tau^{*}$,

$$
\breve{A}=\left(\begin{array}{cc}
A & 0 \\
C^{*} \tilde{C} & A_{W}^{*}
\end{array}\right), \quad \breve{B}=\left(\begin{array}{l}
B \\
0
\end{array}\right), \quad \breve{C}=\left(\begin{array}{ll}
0 & -B^{*}
\end{array}\right),
$$

and $\mathcal{D}(\breve{A})=\mathcal{D}(A) \oplus \mathcal{D}\left(A_{W}^{*}\right)$. Applying Theorem 5.1 to the extended PS - realizations $\theta=(A, B, C ; V, W ; Y)$ and $\theta^{*}=\left(A_{W}^{*}, C^{*}, B^{*} ; W, V ; Y\right)$ one sees that

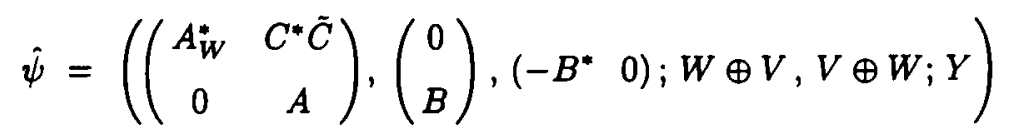

is an extended PS-realization and thus $\psi$ is an extended PS-realization. Further, the operator $\widetilde{C} \in \mathcal{L}(\mathcal{D}(\breve{A}), Y)$ as defined in terms of $\psi$ in Lemma 3.1 is given by

$$
\widetilde{C}=\left(\begin{array}{ll}
0 & -\widetilde{B^{*}}
\end{array}\right),
$$

where $\widetilde{B^{*}} \in \mathcal{L}\left(\mathcal{D}\left(A_{W}^{*}\right), Y\right)$ is defined in terms of $\theta^{*}$ as in Lemma 3.1. Note that $\widetilde{B^{*}}=(\hat{B})^{*} A_{W}^{*}: \mathcal{D}\left(A_{W}^{*}\right) \rightarrow Y$ [cf. (3.5) applied to $\theta^{*}$, and (4.9)]. The resolvent of $\breve{A}$ has the form

$$
(\lambda-\check{A})^{-1}=\left(\begin{array}{cc}
(\lambda-A)^{-1} & 0 \\
\left(\lambda-A_{W}^{*}\right)^{-1} C^{*} \tilde{C}(\lambda-A)^{-1} & \left(\lambda-A_{W}^{*}\right)^{-1}
\end{array}\right) .
$$


Further, $(\breve{A})_{W}$ is most easily described by specifying its resolvent, which is found by using the identity

$$
(\lambda-\breve{A})^{-1} \breve{\tau}=\check{\tau}\left(\lambda-(\breve{A})_{\breve{W}}\right)^{-1}
$$

and is given by

$$
\left(\lambda-(\breve{A})_{W}\right)^{-1}=\left(\begin{array}{cc}
\left(\lambda-A_{W}\right)^{-1} & 0 \\
A^{*}\left(\lambda-A^{*}\right)^{-1} \hat{C}^{*} C\left(\lambda-A_{W}\right)^{-1} & \left(\lambda-A^{*}\right)^{-1}
\end{array}\right)
$$

where we have employed (4.9). Using (5.15) we easily obtain the transfer function

$$
\begin{aligned}
W_{\psi}(\lambda) & =I+\widetilde{C}(\lambda-\breve{A})^{-1} \breve{B} \\
& =I-\left(\begin{array}{cc}
0 & \widetilde{B^{*}}
\end{array}\right)\left(\begin{array}{cc}
\lambda-A & 0 \\
-C^{*} \tilde{C} & \lambda-A_{W}^{*}
\end{array}\right)^{-1}\left(\begin{array}{l}
B \\
0
\end{array}\right) \\
& =I-\widetilde{B^{*}}\left(\lambda-A_{W}^{*}\right)^{-1} C^{*} \tilde{C}(\lambda-A)^{-1} B \\
& =I-\left[W_{\theta}(\lambda)-I\right]\left[W_{\theta}(\lambda)-I\right] .
\end{aligned}
$$

For later use we define

$$
\breve{A}^{\times}=\breve{A}-\breve{B} \widetilde{C}=\left(\begin{array}{cc}
A & B \widetilde{B^{*}} \\
C^{*} \tilde{C} & A_{W}^{*}
\end{array}\right)
$$

with domain $\mathcal{D}(\breve{A})=\mathcal{D}(A) \oplus \mathcal{D}\left(A_{W}^{*}\right)$. Observe that its part $\left(\breve{A}^{\times}\right)_{\mathscr{W}}$ in $\breve{W}$ is not easily specified.

Part (b). Suppose (5.13) is true. Because

$$
\sup _{|\operatorname{Im} \lambda| \leq \nu_{0}}\left\|W_{\psi}(\lambda)-I\right\| \leq\left(\sup _{|\operatorname{Im} \lambda| \leq \nu_{0}}\left\|W_{\theta}(\lambda)-I\right\|\right)^{2}<1,
$$

$W_{\psi}(\cdot)$ has a left quasi-canonical factorization and hence

$$
\breve{V}_{-} \oplus \breve{V}_{+}^{\times}=\breve{V}, \quad \breve{W}_{-} \oplus \breve{W}_{+}^{\times}=\breve{W} .
$$

Since $\sigma(A) \subset \mathbb{C}_{-}$and $\sigma\left(A_{W}^{*}\right) \subset \mathbb{C}_{+},(5.15)$ implies $\breve{V}_{-}=\{0\} \oplus W$. In the same way, since $\sigma\left(A_{W}\right) \subset \mathbb{C}_{-}$and $\sigma\left(A^{*}\right) \subset \mathbb{C}_{+},(5.16)$ implies $\breve{W}_{-}=\{0\} \oplus V$. Hence, there exist $X_{W V} \in \mathcal{L}(V, W)$ and $X_{V W} \in \mathcal{L}(W, V)$ such that

$$
\breve{V}_{+}^{\times}=\operatorname{Im}\left(\begin{array}{c}
I \\
i X_{W V}
\end{array}\right), \quad \breve{W}_{+}^{\times}=\operatorname{Im}\left(\begin{array}{c}
I \\
i X_{V W}
\end{array}\right) \text {. }
$$

Because for every $w \in W$ there exists $v \in V$ such that

$$
\check{\tau}\left(\begin{array}{c}
I \\
i X_{V W}
\end{array}\right) w=\left(\begin{array}{c}
I \\
i X_{W V}
\end{array}\right) v
$$

we find 


$$
\tau^{*} X_{V W}=X_{W V} \tau \text {. }
$$

Thus, noting that $\mathcal{D}\left(\breve{A}^{\times}\right)=\mathcal{D}(\breve{A})$, we get from $\breve{A}^{\times}\left[\mathcal{D}(\breve{A}) \cap \breve{V}_{+}^{\times}\right] \subset \breve{V}_{+}^{\times}$that

$$
\breve{A}^{\times}\left(\begin{array}{c}
I \\
i X_{W V}
\end{array}\right)=\left(\begin{array}{c}
I \\
i X_{W V}
\end{array}\right) A_{0}
$$

for some operator $A_{0}(V \rightarrow V)$. As a result, $\mathcal{D}\left(A_{0}\right)=\mathcal{D}(A), A_{0}=A+i B \widetilde{B}^{*} X_{W V}$, and

$$
C^{*} \tilde{C}+i A_{W}^{*} X_{W V}=i X_{W V}\left(A+i B \widetilde{B^{*}} X_{W V}\right) \text {, }
$$

which implies (5.14). Note that $\left(I i X_{W V}\right)^{T}$ maps $\mathcal{D}\left(A_{0}\right)=\mathcal{D}(A)$ into $\mathcal{D}\left(\breve{A}^{\times}\right)=$ $\mathcal{D}(A) \oplus \mathcal{D}\left(A_{W}^{*}\right)$ and therefore $X_{W V}[\mathcal{D}(A)] \subset \mathcal{D}\left(A_{W}^{*}\right)$. Further, $\sigma\left(A_{0}\right) \subset \mathbb{C}_{-}$, as $A_{0}$ is similar to the part of $\breve{A}^{\times}$in $\breve{V}_{+}^{\times}$.

Let us now consider the identity

$$
\left(-i X_{V W} I\right) \check{A}^{\times}=A_{1, W}^{*}\left(-i X_{V W} I\right)
$$

for some $A_{1, W}^{*}(W \rightarrow W)$. Then $\mathcal{D}\left(A_{1, W}^{*}\right)=\mathcal{D}\left(A_{W}^{*}\right), A_{1, W}^{*}$ is similar to the part of $\breve{A}^{\times}$in $\breve{V}_{-}^{\times}$, and hence $\sigma\left(A_{1, W}^{*}\right) \subset \mathbb{C}_{+}$. We easily find that $A_{1, W}^{*}=A_{W}^{*}-i X_{V W} B \widetilde{B}^{*}$ and (5.14) is satisfied.

Now $X=X_{W V}$ has all the properties required by Theorem 5.4, except possibly $X_{V W}=\left(X_{W V}\right)^{*}$ which will be proved below.

Part (c). Conversely, let $X$ be a solution of (5.14) with the properties mentioned in Theorem 5.4 (except for the selfadjointness of $X \tau$ ). Then

$$
\begin{aligned}
& W_{\psi}(\lambda) \\
= & I-\widetilde{B^{*}}\left(\lambda-A_{W}^{*}\right)^{-1}\left[-i X(\lambda-A)+i\left(\lambda-A_{W}^{*}\right) X-X B \widetilde{B^{*}} X\right](\lambda-A)^{-1} B \\
= & {\left[I+i \widetilde{B}^{*}\left(\lambda-A_{W}^{*}\right)^{-1} X B\right]\left[I-i \widetilde{B}^{*} X(\lambda-A)^{-1} B\right] } \\
= & {\left[I+i \hat{B}^{*} A_{W}^{*}\left(\lambda-A_{W}^{*}\right)^{-1} X B\right]\left[I-i \hat{B}^{*} A_{W}^{*} X(\lambda-A)^{-1} B\right], }
\end{aligned}
$$

where (3.5) applied to $\theta^{*}$ and (4.9) were employed. Then, inverting the two factors in (5.19), we find

$$
W_{\psi}(\lambda)^{-1}=\left[I+i \widetilde{B^{*}} X\left(\lambda-A_{0}\right)^{-1} B\right]\left[I-i \widetilde{B^{*}}\left(\lambda-A_{1, W}^{*}\right)^{-1} X B\right],
$$

where $A_{0}=A+i B \widetilde{B^{*}} X$ satisfies $\sigma\left(A_{0}\right) \subset \mathbb{C}_{-}$and $A_{1, W}^{*}=A_{W}^{*}-i X B \widetilde{B^{*}}$ satisfies $\sigma\left(A_{1, W}^{*}\right) \subset \mathbb{C}_{+}$. Also, $\sigma(A) \subset \mathbb{C}_{-}$and $\sigma\left(A_{W}^{*}\right) \subset \mathbb{C}_{+}$. Thus (5.19) is a right quasi canonical factorization of $W_{\psi}$ and its factors and their inverses tend to the identity operator in the strong operator topology as $\lambda \rightarrow \infty$ in $\overline{\mathbb{C}^{ \pm}}$.

Note from (5.17) that $W_{\psi}$ takes selfadjoint values on the real line. Taking adjoints in (5.18) it easily follows that

$$
W_{\psi}(\lambda)=\left[I+i B^{*}\left(\lambda-A^{*}\right)^{-1} X^{*} A_{W} \hat{B}\right]\left[I-i B^{*} X^{*}\left(\lambda-A_{W}\right)^{-1} A_{W} \hat{B}\right]
$$

is also a right quasi-canonical factorization of $W_{\psi}$, for which the factors tend to the identity operator in the strong operator topology as $\lambda \rightarrow \infty$ in $\overline{\mathbb{C}^{ \pm}}$. However, such a 
right quasi-canonical factorization is readily seen to be unique. Thus for $\lambda \in \mathbb{R}$ the two factors in (5.19) are each others adjoints and hence $W_{\psi}(\lambda) \geq 0$ for all $\lambda \in \mathbb{R}$. But then

$$
\sup _{\lambda \in \mathbb{R}}\left\|W_{\theta}(\lambda)-I\right\|<1 .
$$

Since $W_{\theta}(\cdot)^{-1}$ is bounded on all strips $|\operatorname{Im} \lambda| \leq \nu_{0}$ with $\nu_{0}$ small enough,

$$
\sup _{|\operatorname{Im} \lambda| \leq \nu_{0}}\left\|W_{\theta}(\lambda)-I\right\|<1
$$

for $\nu_{0}$ small enough.

Part (d). It remains to prove that $X \tau \in \mathcal{L}(W)$ is selfadjoint. Using that the factors in (5.19) are each others adjoints, we obtain

$$
\begin{aligned}
W_{\psi}(\lambda) & =\left[I+i B^{*}\left(\lambda-A^{*}\right)^{-1} X^{*} A_{W} \hat{B}\right]\left[I-i B^{*} X^{*} A_{W}\left(\lambda-A_{W}\right)^{-1} \hat{B}\right] \\
& =\left[I+i \widetilde{B}^{*}\left(\lambda-A_{W}^{*}\right)^{-1} X_{V W}^{*} B\right]\left[I-i \widetilde{B}^{*} X_{V W}^{*}(\lambda-A)^{-1} B\right],
\end{aligned}
$$

where we used (4.9) and (5.7). The result is (5.19) with $X$ replaced by $X_{V W}^{*}$. Taking inverses of the factors we find

$$
W_{\psi}(\lambda)^{-1}=\left[I+i \widetilde{B^{*}} X_{V W}^{*}\left(\lambda-A_{1}\right)^{-1} B\right]\left[I-i \widetilde{B^{*}}\left(\lambda-A_{0, W}^{*}\right)^{-1} X_{V W}^{*} B\right],
$$

where $A_{1}=A+i B \widetilde{B^{*}} X_{V W}^{*}$ satisfies $\mathcal{D}\left(A_{1}\right)=\mathcal{D}(A)$ and $\sigma\left(A_{1}\right) \subset \mathbb{C}$ - and $A_{0, W}^{*}=$ $A_{W}^{*}-i X_{V W}^{*} B \widetilde{B^{*}}$ satisfies $\mathcal{D}\left(A_{0, W}^{*}\right)=\mathcal{D}\left(A_{W}^{*}\right)$ and $\sigma\left(A_{0, W}^{*}\right) \subset \mathbb{C}_{+}$. This implies

$$
\breve{A}^{\times}\left(\begin{array}{c}
I \\
i X_{V W}^{*}
\end{array}\right)=\left(\begin{array}{c}
I \\
i X_{V W}^{*}
\end{array}\right) A_{1}
$$

on $\mathcal{D}\left(A_{1}\right)=\mathcal{D}(A)$. Hence $\operatorname{Im}\left(I \quad i X_{V W}^{*}\right)^{T}$ is an $\breve{A}^{\times}$-invariant subspace of $V \oplus W$ and the restriction of $\breve{A}^{\times}$to this subspace has its spectrum contained in $\mathbb{C}$.. Thus

$$
\operatorname{Im}\left(\begin{array}{c}
I \\
i X_{V W}^{*}
\end{array}\right) \subset \operatorname{Im}\left(\begin{array}{c}
I \\
i X
\end{array}\right)=\breve{V}_{+}^{\times}
$$

which implies $X_{V W}^{*}=X$. The selfadjointness of $X \tau$ then follows from (5.18).

We note that

$$
\breve{A}^{\times}\left(\begin{array}{cc}
0 & \tau \\
\tau^{*} & 0
\end{array}\right) \text { and }\left(\check{A}^{\times}\right)_{W}\left(\begin{array}{cc}
0 & \tau^{*} \\
\tau & 0
\end{array}\right)
$$

are selfadjoint operators on $V \oplus W$ and $W \oplus V$, respectively. Thus the spectra of $\breve{A}^{\times}$ and $\left(\breve{A}^{\times}\right)_{W}$ are symmetric with respect to the real axis.

\section{Left and right quasi-canonical factorization}

In this section we give necessary and sufficient conditions for the existence of a right quasi - canonical factorization of the transfer function $W_{\theta}$ of an extended PSrealization if $W_{\theta}$ is assumed to have a left quasi-canonical factorization. 
Lemma 6.1. Let $\theta_{ \pm}=\left(A_{ \pm}, B_{ \pm}, C_{ \pm} ; V_{ \pm}, W_{ \pm} ; Y\right)$ be two extended $P S-$ realizations such that $\sigma\left(A_{ \pm}\right) \cup \sigma\left(A_{ \pm, W}\right) \subset \mathbb{C}_{\mp}$. Then there exists a unique pair $\left(Q, Q_{W}\right)$ of solutions of the Lyapunov equations

$$
\begin{aligned}
A_{+} Q x-Q_{-} x & =-B_{+} \tilde{C}_{-} x, \quad x \in \mathcal{D}\left(A_{-}\right) ; \\
Q_{W} x-A_{+, W}^{-1} Q_{W} A_{-, W} x & =-\hat{B}_{+} C_{-} x, \quad x \in \mathcal{D}\left(A_{-, W}\right),
\end{aligned}
$$

where $Q \in \mathcal{L}\left(V_{-}, V_{+}\right), Q_{W} \in \mathcal{L}\left(W_{-}, W_{+}\right), Q\left[\mathcal{D}\left(A_{-}\right)\right] \subset \mathcal{D}\left(A_{+}\right)$, and $\tilde{C}_{ \pm}$and $\hat{B}_{ \pm}$are defined in terms of the extended PS-realizations $\theta_{ \pm}$in Proposition 3.3 and in (3.5), respectively. This pair of solutions is given by

$$
\begin{aligned}
Q_{x} & =-i \int_{0}^{\infty} e^{-i s A_{+}} B_{+} \tilde{C}_{-} e^{i s A_{-}} x d s, & x \in \mathcal{D}\left(A_{-}\right) \\
Q_{W} x & =-i \tau_{+}^{-1} \int_{0}^{\infty} e^{-i \Delta A_{+}} B_{+} C_{-} e^{i s A_{-}, w} x d s, & x \in \mathcal{D}\left(A_{-}, W\right)
\end{aligned}
$$

Moreover, $Q \tau x=\tau Q_{W} x$ for $x \in \mathcal{D}\left(A_{-, W}\right)$.

Proof. Let $\left(Q, Q_{W}\right)$ be a solution. Then replace $x$ by $e^{i s A}-x$ with $s>0$ in (6.1) while using the invariance of $\mathcal{D}\left(A_{-}\right)$under $e^{i s A_{-}}$, and premultiply the whole expression by $i e^{-i s A_{+}}$while using the invariance of $\mathcal{D}\left(A_{+}\right)$under $e^{-i \varepsilon A_{+}}$, yielding

$$
i\left\{e^{-i s A_{+}} A_{+} Q e^{i \Delta A_{-}} x-e^{-i s A_{+}} Q A_{-} e^{i s A_{-}} x\right\}=-i e^{-i \delta A_{+}} B_{+} \tilde{C}_{-} e^{i \theta A_{-}} x,
$$

where $x \in \mathcal{D}\left(A_{-}\right)$. Hence for $r>0$ we have

$$
Q x-e^{-i r A+} Q e^{i r A}-x=-i \int_{0}^{r} e^{-i \varepsilon A_{+}} B_{+} \tilde{C}_{-} e^{i \varepsilon A-} x d s,
$$

so that

$$
Q x=-i \int_{0}^{\infty} e^{-i s A_{+}} B_{+} \tilde{C}_{-} e^{i \varepsilon A_{-}} x d s
$$

Thus

$$
Q x=-i \tau_{+} \Gamma_{\theta_{+}} \Lambda_{\theta_{-}} x, \quad x \in \mathcal{D}\left(A_{-}\right),
$$

and we may conclude that $Q$ extends uniquely to an operator in $\mathcal{L}\left(V_{-}, V_{+}\right)$.

In a similar manner, let $x \in \mathcal{D}\left(A_{-, W}\right)$ satisfy (6.2). Then $\tau_{-} x \in \mathcal{D}\left(A_{-}\right)$and

$$
-A_{+}^{-1} B_{+} \tilde{C}_{-} \tau_{-} x=-\tau_{+} \hat{B}_{+} C_{-} x=\tau_{+} Q_{W} x-A_{+}^{-1} \tau_{+} Q_{W} A_{-, W} x,
$$

so that $\tau_{+} Q_{W} x \in \mathcal{D}\left(A_{+}\right)$. Then $A_{+} \tau_{+} Q_{W} x=-B_{+} \bar{C}_{-} \tau_{-} x+\tau_{+} Q_{W} A_{-, W} x$ and therefore $Q_{W} x \in \mathcal{D}\left(A_{+, W}\right)$, and hence

$$
A_{+} \tau_{+} Q_{W} x-\tau_{+} Q_{W} A_{-, W} x=-B_{+} C_{-} x .
$$

Then replacing $x$ by $e^{i s A_{-, w}} x$ with $s>0$, premultiplying the resulting expression by $i e^{-i s A_{+}}$, we obtain by integration

$$
\tau_{+} Q_{W} x-e^{-i r A_{+}} \tau_{+} Q_{W} e^{i r A_{-, W}} x=-i \int_{0}^{r} e^{-i s A_{+}} B_{+} C_{-} e^{i s A_{-, W}} x d s,
$$


which implies (6.4) for $x \in \mathcal{D}\left(A_{-,}\right.$, $)$. Observing that

$$
Q_{W} x=-i \Gamma_{\theta_{+}} \Lambda_{\theta_{-}} \tau_{-} x, \quad x \in \mathcal{D}\left(A_{-}, W\right)
$$

we get (6.4).

Conversely, if $\left(Q, Q_{W}\right)$ is given by (6.3) and (6.4), the Lyapunov equations (6.1) and (6.2) are immediate. Further, $\tau_{+} Q_{W} x=Q \tau x$ for $x \in \mathcal{D}\left(A_{-, W}\right)$.

Theorem 6.2. Let $\theta_{ \pm}=\left(A_{ \pm}, B_{ \pm}, C_{ \pm} ; V_{ \pm}, W_{ \pm} ; Y\right)$ be two extended $P S$ - realizations with $\sigma\left(A_{ \pm}\right) \cup \sigma\left(A_{ \pm, W}\right) \subset \mathbb{C}_{\mp}$, and let $\theta=(A, B, C ; V, W ; Y)$ be the product of $\theta_{+}$and $\theta_{-}$. In particular,

$$
W_{\theta}(\lambda)=W_{\theta_{+}}(\lambda) W_{\theta_{-}}(\lambda), \quad \lambda \in \mathbb{R},
$$

is a left quasi-canonical factorization. Let $\left(Q, Q_{W}\right)$ be the unique pair of solutions of the Lyapunov equations (6.1), (6.2), i.e.,

$$
\begin{aligned}
A_{+} Q x-Q_{-} x & =-B_{+} \tilde{C}_{-} x, & & x \in \mathcal{D}\left(A_{-}\right) ; \\
Q_{W} x-A_{+, W}^{-1} Q_{W} A_{-, W} x & =-\hat{B}_{+} C_{-} x, & & x \in \mathcal{D}\left(A_{-, W}\right),
\end{aligned}
$$

and let $\left(P, P_{W}\right)$ be the unique pair of solutions of the Lyapunov equations

$$
\begin{aligned}
A_{-}^{\times} P x-P A_{+}^{\times} x & =B_{-} \tilde{C}_{+} x, \quad x \in \mathcal{D}\left(A_{+}^{\times}\right)=\mathcal{D}\left(A_{+}\right) ; \\
P_{W} x-\left(A_{-, W}^{\times}\right)^{-1} P_{W} A_{+, W}^{\times} x & =\hat{B}_{-}^{\times} C_{+} x, \quad x \in \mathcal{D}\left(A_{+, W}^{\times}\right)
\end{aligned}
$$

where $\bar{C}_{ \pm}$and $\hat{B}_{+}$are defined in terms of the extended PS-realizations $\theta_{ \pm}$in Proposition 3.3 and in (3.5), and $\hat{B}_{-}^{\times}=\tau_{-}^{-1}\left(A_{-}^{\times}\right)^{-1} B_{-} \in \mathcal{L}\left(Y, W_{-}\right)$. Then $W_{\theta}$ has a right quasi-canonical factorization

$$
W_{\theta}(\lambda)=W_{\theta_{m}}(\lambda) W_{\theta_{p}}(\lambda), \quad \lambda \in \mathbb{R}
$$

if and only if the operator $I-Q P$ is boundedly invertible on $V_{+}$and the operator $I-Q_{W} P_{W}$ is boundedly invertible on $W_{+}$, or, equivalently, if and only if the operator $I-P Q$ is boundedly invertible on $V_{-}$and the operator $I-P_{W} Q_{W}$ is boundedly invertible on $W_{-}$. When this is the case, the factors $W_{\theta_{m}}$ and $W_{\theta_{p}}$ are given by the formulas

$$
\begin{aligned}
& W_{\theta_{m}}(\lambda)=I+\left(\tilde{C}_{+} Q+\tilde{C}_{-}\right)\left(\lambda-A_{-}\right)^{-1}(I-P Q)^{-1}\left(-P B_{+}+B_{-}\right) \\
& W_{\theta_{p}}(\lambda)=I+\left(\tilde{C}_{+}+\tilde{C}_{-} P\right)(I-Q P)^{-1}\left(\lambda-A_{+}\right)^{-1}\left(B_{+}-Q B_{-}\right)
\end{aligned}
$$

and their inverses are given by

$$
\begin{aligned}
& W_{\theta_{m}}(\lambda)^{-1}=I-\left(\tilde{C}_{+} Q_{W}+\tilde{C}_{-}\right)(I-P Q)^{-1}\left(\lambda-A_{-}^{\times}\right)^{-1}\left(-P B_{+}+B_{-}\right) \\
& W_{\theta_{p}}(\lambda)^{-1}=I-\left(\tilde{C}_{+}+\tilde{C}_{-} P\right)\left(\lambda-A_{+}^{x}\right)^{-1}(I-Q P)^{-1}\left(B_{+}-Q B_{-}\right)
\end{aligned}
$$

Proof. We split the proof into three parts. 
Part (a). Let $\theta=(A, B, C ; V, W ; Y)$ be the extended PS-realization with transfer function $W_{\theta}$ constructed as the product of $\theta_{+}$and $\theta_{-}$. Then, by Theorem 4.1,

$$
A=\left(\begin{array}{cc}
A_{+} & B_{+} \tilde{C}_{-} \\
0 & A_{-}
\end{array}\right), \quad B=\left(\begin{array}{c}
B_{+} \\
B_{-}
\end{array}\right), \quad C=\left(C_{+} C_{-}\right), \quad \tau=\tau_{+} \oplus \tau_{-},
$$

as well as

$$
E(t ;-i A)=\left(\begin{array}{cc}
E\left(t ;-i A_{+}\right) & F(t) \\
0 & E\left(t ;-i A_{-}\right)
\end{array}\right)
$$

with

$$
F(t) x=-i \int_{-\infty}^{\infty} E\left(t-s ;-i A_{+}\right) B_{+}\left(\Lambda_{\theta_{-}} x\right)(s) d s, \quad x \in V_{-},
$$

and

$$
E\left(t ;-i A_{W}\right)=\left(\begin{array}{cc}
E\left(t ;-i A_{+, W}\right) & F_{W}(t) \\
0 & E\left(t ;-i A_{-, W}\right)
\end{array}\right)
$$

with

$$
F_{W}(t) x=-i \tau_{+}^{-1} \int_{-\infty}^{\infty} E\left(t-s ;-i A_{+, W}\right) \hat{B}_{+} C_{-} E\left(s ;-i A_{-, W}\right) x d s, \quad x \in W_{-} .
$$

By the same token, if we depart from the factorization $\theta^{\times}=\theta_{-}^{\times} \theta_{+}^{\times}$, we find in addition

$$
A^{\times}=\left(\begin{array}{cc}
A_{+}^{\times} & 0 \\
-B_{-} \widetilde{C_{+}} & A_{-}^{\times}
\end{array}\right) \text {, }
$$

as well as

$$
E\left(t ;-i A^{\times}\right)=\left(\begin{array}{cc}
E\left(t ;-i A_{+}^{\times}\right) & 0 \\
F^{\times}(t) & E\left(t ;-i A_{-}^{\times}\right)
\end{array}\right)
$$

with

$$
F^{\times}(t) x=i \int_{-\infty}^{\infty} E\left(t-s ;-i A_{+}^{\times}\right) B_{+}\left(\Lambda_{\theta_{-}} x\right)(s) d s, \quad x \in V_{-},
$$

and

$$
E\left(t ;-i A_{W}^{\times}\right)=\left(\begin{array}{cc}
E\left(t ;-i A_{+, W}^{\times}\right) & 0 \\
F_{W}^{\times}(t) & E\left(t ;-i A_{-, W}^{\times}\right)
\end{array}\right),
$$

with

$$
F_{W}^{\times}(t) x=i \tau_{+}^{-1} \int_{-\infty}^{\infty} E\left(t-s ;-i A_{+, W}^{\times}\right) \hat{B}_{+} C_{-} E\left(s ;-i A_{-, W}^{\times}\right) x d s, \quad x \in W_{-} .
$$

Now note that there is

(a) a closed $A$-invariant subspace of $V$ complementary to $V_{+} \oplus(0)$, namely the spectral subspace of $A$ with respect to its spectrum in $\mathbb{C}_{-}$.

Likewise, there exist

(b) a closed $A_{W}$ - invariant subspace of $W$ complementary to $W_{+} \oplus(0)$,

(c) a closed $A^{\times}$-invariant subspace of $V$ complementary to (0) $\oplus V_{-}^{\mathrm{X}}$, and

(d) a closed $A_{W}^{\times}$-invariant subspace of $W$ complementary to $(0) \oplus W_{-}^{\times}$.

Thus there exist 
(a) $Q \in \mathcal{L}\left(V_{-}, V_{+}\right)$such that $\left(\begin{array}{c}Q \\ I_{V_{-}}\end{array}\right)$leaves invariant $\mathcal{D}(A)$ and is $A$-invariant,

(b) $Q_{W} \in \mathcal{L}\left(W_{-}, W_{+}\right)$such that $\left(\begin{array}{c}Q_{W} \\ I_{W_{-}}\end{array}\right)$leaves invariant $\mathcal{D}\left(A_{W}\right)$ and is $A_{W}-$ invariant,

(c) $P \in \mathcal{L}\left(V_{+}, V_{-}\right)$such that $\left(\begin{array}{c}I_{V_{+}} \\ P\end{array}\right)$ leaves invariant $\mathcal{D}\left(A^{\times}\right)=\mathcal{D}(A)$ and is $A^{\times}-$ invariant, and

(d) $P_{W} \in \mathcal{L}\left(W_{+}, W_{-}\right)$such that $\left(\begin{array}{c}I_{W_{+}} \\ P_{W}\end{array}\right)$ leaves invariant $\mathcal{D}\left(A_{W}^{\times}\right)$and is $A_{W^{-}}^{\times}$ invariant.

Further, $Q \tau_{-}=\tau_{+} Q_{W}$ and $P \tau_{+}=\tau_{-} P_{W}$. Moreover, since

$$
\mathcal{D}(A)=\mathcal{D}\left(A^{\times}\right)=\mathcal{D}\left(A_{+}\right) \oplus \mathcal{D}\left(A_{-}\right)=\mathcal{D}\left(A_{+}^{\times}\right) \oplus \mathcal{D}\left(A_{-}^{\times}\right),
$$

we have $Q\left[\mathcal{D}\left(A_{-}\right)\right] \subset \mathcal{D}\left(A_{+}\right)$and $P\left[\mathcal{D}\left(A_{+}\right)\right] \subset \mathcal{D}\left(A_{-}\right)$. Furthermore, the Lyapunov equations (6.1) and (6.5) are satisfied. The identities $Q \tau_{-}=\tau_{+} Q_{W}$ and $P \tau_{+}=\tau_{-} P_{W}$ then imply that the Lyapunov equations (6.2) and (6.6) are satisfied as well.

Part (b). Now suppose $W_{\theta}$ has a right quasi-canonical factorization. Then, by Theorem 5.2, the ranges of $\left(\begin{array}{c}Q \\ I_{V_{-}}\end{array}\right)$and $\left(\begin{array}{c}I_{V_{+}} \\ P\end{array}\right)$ have a trivial intersection and add up to $V$, and the ranges of $\left(\begin{array}{c}Q_{W} \\ I_{W_{-}}\end{array}\right)$and $\left(\begin{array}{c}I_{W_{+}} \\ P_{W}\end{array}\right)$ have a trivial intersection and add up to $W$. Consequently, $I-P Q, I-Q P, I-P_{W} Q_{W}$ and $I-Q_{W} P_{W}$ are boundedly invertible on $V_{-}, V_{+}, W_{-}$, and $W_{+}$, respectively.

Part (c). Conversely, let the operators $Q \in \mathcal{L}\left(V_{-}, V_{+}\right), Q_{W} \in \mathcal{L}\left(W_{-}, W_{+}\right), P \in$ $\mathcal{L}\left(V_{+}, V_{-}\right)$and $P_{W} \in \mathcal{L}\left(W_{+}, W_{-}\right)$be as in Part (a), and assume that $I-Q P$ is boundedly invertible on $V_{+}$, and $I-Q_{W} P_{W}$ is boundedly invertible on $W_{+}$. Then

$$
\operatorname{Im}\left(\begin{array}{c}
Q \\
I_{V_{-}}
\end{array}\right) \oplus \operatorname{Im}\left(\begin{array}{c}
I_{V_{+}} \\
P
\end{array}\right)=V, \quad \operatorname{Im}\left(\begin{array}{c}
Q_{W} \\
I_{W_{-}}
\end{array}\right) \oplus \operatorname{Im}\left(\begin{array}{c}
I_{W_{+}} \\
P_{W}
\end{array}\right)=W
$$

We then define the projection $\Pi$ of $V$ onto $\operatorname{Im}\left(\begin{array}{c}I_{V_{+}} \\ P\end{array}\right)$ along $\operatorname{Im}\left(\begin{array}{c}Q \\ I_{V_{-}}\end{array}\right)$and the projection $\Pi_{W}$ of $W$ onto $\operatorname{Im}\left(\begin{array}{c}I_{W_{+}} \\ P_{W}\end{array}\right)$ along $\operatorname{Im}\left(\begin{array}{c}Q_{W} \\ I_{W_{-}}\end{array}\right)$. It is easily seen that

$$
\begin{aligned}
& \Pi=\left(\begin{array}{c}
I_{V_{+}} \\
P
\end{array}\right)(I-Q P)^{-1}\left(I_{V_{+}}-Q\right) \\
& \Pi_{W}=\left(\begin{array}{c}
I_{W_{+}} \\
P_{W}
\end{array}\right)\left(I-Q_{W} P_{W}\right)^{-1}\left(I_{W_{+}}-Q_{W}\right) \\
& I-\Pi=\left(\begin{array}{c}
Q \\
I_{V_{-}}
\end{array}\right)(I-P Q)^{-1}\left(-P I_{V_{-}}\right) \\
& I-\Pi_{W}=\left(\begin{array}{c}
Q_{W} \\
I_{W_{-}}
\end{array}\right)\left(I-P_{W} Q_{W}\right)^{-1}\left(\begin{array}{ll}
-P_{W} & I_{W_{-}}
\end{array}\right) .
\end{aligned}
$$


Applying Theorem 5.1 we obtain the right quasi-canonical factorization (6.7), where $\theta_{m}=\left(A_{m}, B_{m}, C_{m} ; V_{m}, W_{m} ; Y\right)$ with $V_{m}=\operatorname{Ker} \Pi, W_{m}=\operatorname{Ker} \Pi_{W}, A_{m}\left(V_{m} \rightarrow V_{m}\right)$ and $C_{m} \in \mathcal{L}\left(W_{m}, Y\right)$ the restrictions of $A$ and $C$ to $V_{m}$ and $W_{m}$, respectively, and $B_{m} \in \mathcal{L}\left(Y, V_{m}\right)$ given by $B_{m} x=(I-I) B x$, and where $\theta_{p}=\left(A_{p}, B_{p}, C_{p} ; V_{p}, W_{p} ; Y\right)$ with $V_{p}=\operatorname{Im} \Pi, W_{p}=\operatorname{Im} \Pi_{W}, A_{p}\left(V_{p} \rightarrow V_{p}\right), C_{p} \in \mathcal{L}\left(W_{p}, Y\right)$ and $B_{p} \in \mathcal{L}\left(Y, V_{p}\right)$ given by $A_{p} x=\Pi A x$ for $x \in V_{p}, C_{p} x=C x$ for $x \in W_{p}$ and $B_{p} x=\Pi B x$. Observe that $V_{m}$ is indeed $A$-invariant because of (6.1), and $V_{p}$ is $A^{\times}$-invariant because of (6.5). Likewise, the invariance of $W_{m}$ under $A_{W}$ follows from (6.2), while the invariance of $W_{p}$ under $A_{W}^{\times}$follows from (6.6).

Let us now define the operators $S \in \mathcal{L}\left(V_{-}, \operatorname{Ker} \Pi\right), S_{W} \in \mathcal{L}\left(W_{-}, \operatorname{Ker} \Pi_{W}\right), T \in$ $\mathcal{L}\left(V_{+}, \operatorname{Im} \Pi\right)$ and $T_{W} \in \mathcal{L}\left(W_{+}, \operatorname{Im} \Pi_{W}\right)$ given by

$$
S=\left(\begin{array}{c}
Q \\
I_{V_{-}}
\end{array}\right), \quad S_{W}=\left(\begin{array}{c}
Q_{W} \\
I_{W_{-}}
\end{array}\right), \quad T=\left(\begin{array}{c}
I_{V_{+}} \\
P
\end{array}\right), \quad T_{W}=\left(\begin{array}{c}
I_{W_{+}} \\
P_{W}
\end{array}\right) .
$$

Then these four operators are boundedly invertible and their inverses are given by

$$
\begin{array}{ll}
S^{-1}=(I-P Q)^{-1}\left(-P I_{V_{-}}\right), & S_{W}^{-1}=\left(I-P_{W} Q_{W}\right)^{-1}\left(-P_{W} I_{W_{-}}\right) ; \\
T^{-1}=(I-Q P)^{-1}\left(I_{V_{+}}-Q\right), & T_{W}^{-1}=\left(I-Q_{W} P_{W}\right)^{-1}\left(I_{W_{+}}-Q_{W}\right) .
\end{array}
$$

Moreover, $S\left[\mathcal{D}\left(A_{-}\right)\right]=\mathcal{D}\left(A_{m}\right)$, and $T\left[\mathcal{D}\left(A_{+}\right)\right]=\mathcal{D}\left(A_{p}\right)$. We find with the help of the four expressions (6.12) $-(6.15)$ and the expressions for $S, T, S^{-1}$ and $T^{-1}$ that

$$
\begin{aligned}
S^{-1} A_{m} S & =A_{-}, & T^{-1} A_{p} T & =(I-Q P)^{-1} A_{+}(I-Q P), \\
S^{-1} B_{m} & =(I-P Q)^{-1}\left(-P B_{+}+B_{-}\right), & T^{-1} B_{p} & =(I-Q P)^{-1}\left(B_{+}-Q B_{-}\right) \\
C_{m} S_{W} & =C_{+} Q_{W}+C_{-} ; & C_{p} T_{W} & =C_{+}+C_{-} P_{W} .
\end{aligned}
$$

Using that $S \tau=\tau S_{W}$ and $T \tau=\tau T_{W}$, we obtain in addition

$$
\begin{aligned}
S_{W}^{-1} A_{m, W} S_{W} & =A_{-, W} \\
T_{W}^{-1} A_{p, W} T & =\left(I-Q_{W} P_{W}\right)^{-1} A_{+, W}\left(I-Q_{W} P_{W}\right) .
\end{aligned}
$$

We thus find the right quasi-canonical factorization (6.7) where the factors are given by (6.8) and (6.9). The factors $(6.10)$ and (6.11) are found by considering $\theta^{\times}=\theta_{p}^{\times} \theta_{m}^{\times}$.

\section{Acknowledgements}

The authors thank R.F. CURTAIN for suggesting the problem of left versus right canonical factorization in the class of Pritchard-Salamon systems. The work of the last two authors was supported by the NATO grant CGR 960700.

\section{References}

[BC] Ball, J. A., and CARPENTER, L.: Realizations of Products and Wiener-Hopf Factors for a Class of Matrix Functions Analytic in a Strip. In: KAASHOEK, M. A., VAN SCHUPPEN, J.H., and Ran, A.C.M. (Eds.), Signal Processing, Scattering and Operator Theory, Proceeding of the MTNS-89, Vol. III, pp. 291-299, Birkhžuser, Boston, 1990 
[BR] BALL, J.A., and RAN, A.C. M.: Left Versus Right Canonical Wiener-Hopf Factorization. In: Gohberg, I., and KAAshoEK, M.A., Constructive Methods of Wiener-Hopf Factorization, Birkhäuser OT 21, pp. 9-38, Basel and Boston, 1986

[BGK1] Bart, H., Gohberg, I., and KaAshozk, M.A.: Minimal Factorization of Matrix and Operator Functions, Birkhäuser OT 1, Basel and Boston, 1979

[BGK2] BART, H., Goheeng, I., and KAAShoek, M. A.: Wiener-Hopf Factorization, Inverse Fourier Transforms and Exponentially Dichotomous Operators, J. Funct. Anal. 68 (1986), $1-42$

[BGK3] BART, H., Gohberg, I., and KAAShozk, M. A.: Exponentially Dichotomous Operators and Inverse Fourier Transforms, Report $8511 / \mathrm{M}$, Econometric Institute, Erasmus University of Rotterdam, The Netherlands, 1985

[C] Curtain, R. F.: Equivalence of Input-Output Stability and Exponential Stability for Infinite-Dimensional Systems, Math. Systems Theory 21 (1988), 19-48

[ClTZ] Curtain, R. F., Logemann, H., Townley, S., and Zwart, H.: Well-Posedness, Stabilizability and Admissibility for Pritchard-Salamon Systems, J. Math. Systems, Estim. Control 4 (1994), 493-496

[CR] Curtain, R. F., and Ran, A. C. M.: Explicit Formulas for Hankel Norm Approximations of Infinite-Dimensional Systems. Integral Equations and Operator Theory 12 (1989), 455469

[CZ1] CURTaIN, R.F., and ZWART, H.: An Introduction to Infinite-Dimensional Linear System Theory, Springer TAM 21, New York, etc., 1995

[C22] Curtain, R. F., and Zwart, H.: The Nehari Problem for the Pritchard-Salamon Clase of Infinite Dimensional Linear Systems: A Direct Approach, Integral Equations and Operator Theory 18 (1994), $130-153$

[DU] Diestel, J., and UhL, J. J., JR.: Vector Measures, Math. Surveys, Vol. 15, Amer. Math. Soc., Providence, R.I., 1977

[GGLD] Glover, K., Green, M., Limebeer, D., and Doyle, J.: A J-Spectral Factorization Approach to $H_{\infty}$ Control, SIAM J. Control and Opt. 28 (1990), 1350-1371

[GGK] · Gohberg, I., Goldberg, S., and KaAshoek, M. A.: Classes of Linear Operators, Vol. I, Birkhäuser OT 49, Basel and Boston, 1990

[GL] Gohberg, 1. C., and LeITerer, J.: Factorization of Operator Functions with respect to a Contour. II. Canonical Factorization of Operators Close to the Identity, Math. Nachrichten 54 (1972), $41-74$ [Russian]

[GRa] Gohberg, I., and Ran, A. C. M.: On Pseudo-Canonical Factorization of Rational Matrix Functions. Indagationes Mathematicae N. S. 4 (1993), 51-63

[GRu] Gohberg, I., and Rubinstein, S.: Proper Contractions and Their Unitary Minimal Completions. In: I. GOHBERG, Ed., Topics in Interpolation Theory of Rational Matrix-Valued Functions, OT s3, $109-122$, Birkhäuser Verlag, Basel, 1988

[GMP] Greeneerg, W., van der Mee, C. V. M., and Protopopescu, V.: Boundary Value Problems in Abstract Kinetic Theory, Birkhäuser OT 23, Basel and Boston, 1987

[HP] Hille, E., and Phillips, R. S.: Functional Analysis, and Semirgoups, Amer. Math. Soc., Providence, R. I., 1957

[KMR] KaAshoek, M.A., VAN DER MEe, C. V.M., and RaN, A. C. M.: Weighting Operator Patterns of Pritchard-Salamon Realizations. Integral Equations and Operator Theory 27 (1997), 497-501

[vK1] VAN Keulen, B.: $\mathcal{H}_{\infty}$-Control for Infinite-Dimensional Systems: A State-Space Approach, Birkhiuser, Boston, 1993

[vK2] vaN KeULEN, B.: Equivalent Conditions for the Solvability of the Nonstandard LQProblem for Pritchard-Salamon Systems, SIAM J. Control and Opt. 33 (1995), 13261356 
[P] PAzY, A.: Semigroups of Linear Operators and Applications to Partial Differential Equations, Applied Mathematical Sciences, Vol. 44, Springer-Verlag, New York, etc., 1983

[PS] Prutchard, A.J., and SAlamon, D.: The Linear Quadratic Optimal Control Problem for Infinite Dimensional Systems with Unbounded Input and Output Operators, SIAM J. Control and Opt. 25 (1987), $121-144$

[R] RAN, A.C. M.: Hankel-Norm Approximation for Infinite Dimensional Systems and Wiener-Hopf Factorization. In: R.F. CurTaIN, Ed., Modelling, Robustness and Sensitivity Reduction in Control Systems, 57-69, Springer-Verlag, Berlin, etc., 1987

[S] SAlAMON, D.: Control and Observation of Neutral Systems. Research Notes in Mathematics, Vol. 91, Pitman, Boston, 1984

M.A. Koashoek and A.C.M. Ran Faculteit Wiskunde en Informatica Vrije Universiteit

De Boelelaan 1081a

$1081 \mathrm{HV}$ Amstendam

The Netherlands

kaashOcs.vu.nl and ranOcs.vu.nl
C. V.M. van der Mee

Dipartimento di Matematica

Universitd di Cagliari

Via Ospedale 72

09124 Cagliari

Italy

cormelisOkrein.unica.it 